\title{
Cascaded Metasurface Design Using Electromagnetic Inversion with Gradient-Based Optimization
}

\author{
Trevor Brown, Student Member, IEEE and Puyan Mojabi, Member, IEEE
}

\begin{abstract}
This paper presents an electromagnetic inversion algorithm for the design of cascaded metasurfaces that enables the design process to begin from more practical output field specifications such as a desired power pattern or far-field performance criteria. Thus, this method combines the greater field transformation support of multiple metasurfaces with the flexibility of the electromagnetic inverse source framework. To this end, two optimization problems are formed: one associated with the interior space between two metasurfaces, and the other for the exterior space. The cost functionals corresponding to each of these two optimization problems are minimized using the nonlinear conjugate gradient algorithm with analytic expressions for the gradient operators. A total variation regularizer is incorporated into the optimization procedure to favour smooth field variations from one unit cell to the next. The numerical implementation of the developed design procedure is presented in detail along with several two-dimensional (2D) simulated examples to demonstrate the capabilities of the method.
\end{abstract}

Index Terms-Electromagnetic metasurfaces, inverse problems, inverse source problems, optimization, antenna pattern synthesis.

\section{INTRODUCTION}

$\mathbf{M}$ ETASURFACES offer a level of systematic control over electromagnetic fields not typically possible with conventional materials [1]-[6]. These thin metamaterials of subwavelength thickness can be designed to support arbitrary field discontinuities, leading to applications such as radiation pattern control [7], polarization control [8], [9], impedance matching [10], cloaking [11], and others. Fabrication of these metasurfaces is often much simpler than bulk metamaterials, typically using established printed circuit board (PCB) techniques and materials.

Metasurface design can be decomposed into two distinct steps: macroscopic and mircoscopic [12]. The first step, macroscopic design, is to determine a homogenized representation of the metasurface that is able to effectively model the desired field transformation. Several representations are common in the literature, including effective surface susceptibilities [13], surface impedances/admittances [2], or surface polarizabilities [5]; herein, we adopt the use of surface susceptibilities. The second step, microscopic design, is to determine physical unit cell structures that exhibit the behaviour of the metasurface model. For example, in [14] and [15], three

T. Brown and P. Mojabi are with the Department of Electrical and Computer Engineering, University of Manitoba, Winnipeg, MB, Canada (email: umbrow47@myumanitoba.ca). metallic dogbone layers have been used on two dielectric substrates connected by a bondply layer to implement the desired metasurface model. Microscopic design, although important, is not considered in the work presented here.

Several macroscopic metasurface design methods can be found in recent literature [12], [13]; however, most methods require knowledge of both the incident and the desired transmitted field on the metasurface itself. While the incident field is either known analytically or easily measured, an analytical representation of the transmitted field is typically only possible in ideal cases involving plane waves or other simple fields. We recently presented a macroscopic design method that allows for more practical design criteria, such as far-field (FF) performance criteria (e.g., main beam direction, halfpower beamwidth (HPBW), null locations, etc.) [16], [17]. Since this method is based on solving an electromagnetic inverse source problem, we have referred to this approach as an electromagnetic inversion algorithm for metasurface design. Subsequently, in [18], we modified this inversion method by augmenting its associated cost functional to enforce local power conservation (LPC) [19], [20] which ensures the resulting metasurfaces can be implemented using passive, lossless, and reciprocal elements.

While practically necessary, the drawback of enforcing LPC is that the supported field transformations are restricted. One possible solution is to utilize multiple metasurfaces in succession, as described in [7], [21]-[24]. In this scenario, the field transformations at each metasurface still satisfy LPC, but the transmitted field from the last metasurface no longer has to have the same power distribution as the field incident on the first metasurface. This extra freedom can support a greater variety of output fields by allowing for a redistribution of the incident field power onto the final (output) metasurface. Considering the cascaded metasurfaces as a single structure, total power is still conserved from incident to output fields but the power is redistributed locally.

The design methods presented in [7], [21]-[24] utilize metasurface pairs to perform field transformations that would not be possible with a single metasurface. Similar to the macroscopic design methods mentioned above, these methods require explicit knowledge of the transmitted field on the output metasurface. Therefore, the aim of this paper is to adapt the electromagnetic inversion metasurface design method to the design of cascaded metasurfaces (in our case, two metasurfaces) to allow for more general output field specifications. For example, the design objective can be to meet a specific 
power pattern (phaseless) or to meet performance criteria such as the HPBW and null locations.

To this end, we cast the design problem as two optimization problems. The first optimization problem aims to infer the required tangential fields on the output surface of the second metasurface so as to meet the field specifications. As will be seen, these inferred tangential fields need to satisfy total power conservation (TPC) with respect to the incident power impinging on the first metasurface. The second optimization problem is concerned with finding the tangential fields in the interior space between the two metasurfaces. As will be discussed, these fields need to satisfy LPC for each individual metasurface to ensure that they can be fabricated using passive and lossless elements. It is worth noting that while in [18] we used a stochastic method (particle swarm) to enforce LPC in the inversion process, in this work we use gradient-based optimization for improved convergence. Furthermore, we introduce regularization into the optimization process based on the $L^{2}$-norm total variation (TV) regularizer commonly used for the inverse problem associated with microwave imaging [25], [26]. This regularizer has a smoothing effect on the achievable solution which translates into less field variations from one unit cell of the metasurface to the neighbouring unit cell. This results in several benefits which will be discussed in the paper.

We begin with a description of the problem statement followed by a high-level overview of the proposed methodology. We then explain the numerical implementation of each step of the method in detail. (The derivation of the required gradient operators are provided in the appendices.) This is followed by a series of full-wave simulated examples in both $2 \mathrm{D}$ transverse magnetic and transverse electric cases. Lastly, we identify the existing limitations of the proposed method and present our conclusions.

\section{Problem Statement}

We consider the design of a pair of metasurfaces, denoted respectively as $\Sigma_{1}$ and $\Sigma_{2}$ as shown in Figure 1. In this paper we restrict our discussion to that of planar, parallel metasurfaces separated by a distance $d$, but the theory presented is consistent with more complicated geometries. A known electromagnetic source produces an incident field $\vec{\Psi}^{\text {inc }}$ where $\vec{\Psi} \in\{\vec{E}, \vec{H}\}$ that impinges on the input metasurface, $\Sigma_{1}$. The interaction of this incident field with the pair of metasurfaces will create a reflected field $\vec{\Psi}^{\text {ref }}$ (which may be zero) emanating from $\Sigma_{1}$ and a transmitted field $\vec{\Psi}^{\text {tr }}$ emanating from the output metasurface, $\Sigma_{2}$.

It is important to note that this design method does not require prior knowledge of $\vec{\Psi}^{\text {tr }}$ as is common in alternative methods, but rather a set of user-defined field specifications, denoted as $\mathbf{f}$ on some region of interest $S$. As noted in [17], the field specifications could be provided in any of the following forms:

- complex fields (amplitude and phase information) in either the near-field (NF) or far-field (FF) region,

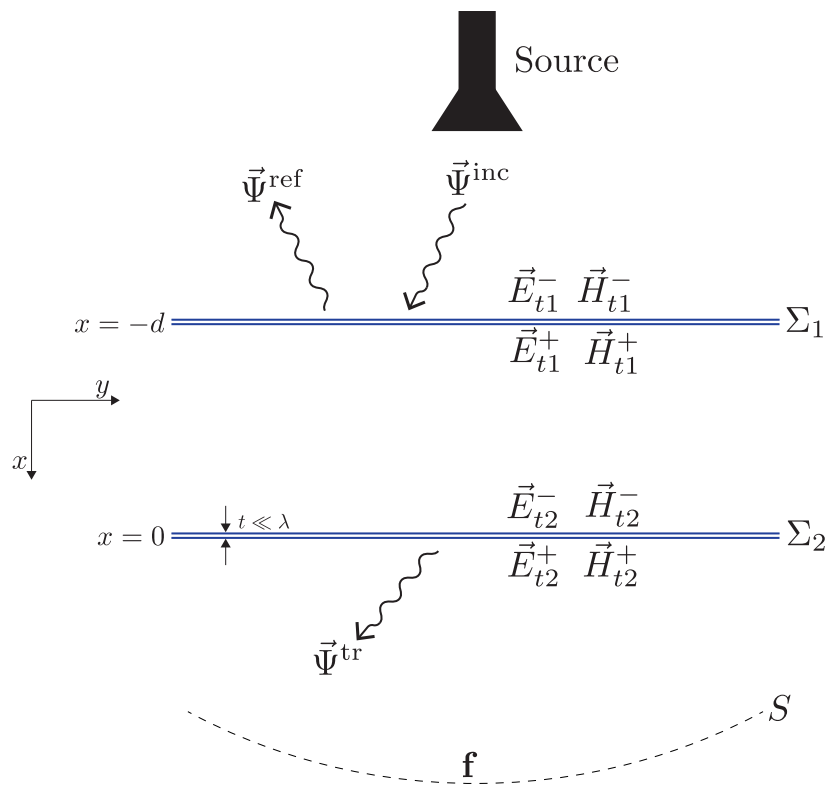

Fig. 1. Overview of the cascaded metasurface design problem. The two metasurfaces are denoted as $\Sigma_{1}$ and $\Sigma_{2}$ and separated by a distance of $d$. The inputs to the design method are the incident field $\vec{\Psi}^{\text {inc }}$ created by the source which impinges on the first metasurface, and the field specifications f defined on the region of interest $S$. The fields $\vec{E}_{t 1}^{-}$and $\vec{H}_{t 1}^{-}$denote the tangential components of the electric and magnetic field on the input side of $\Sigma_{1}$ ('-' superscript), while $\vec{E}_{t 1}^{+}$and $\vec{H}_{t 1}^{+}$represent the tangential fields on the output side of $\Sigma_{1}$ ('+' superscript). The tangential fields on $\Sigma_{2}$ are defined analogously but with a ' 2 ' subscript instead of a ' 1 '. The transmitted and reflected fields produced by the interaction are depicted as $\vec{\Psi}^{\text {tr }}$ and $\vec{\Psi}^{\text {ref }}$, respectively.

- phaseless fields (amplitude-only) in either the NF or FF region $^{1}$, or

- FF performance criteria such as main beam directions, half-power beamwidth (HPBW), and null locations. ${ }^{2}$

Each metasurface is characterized by a set of surface susceptibility distributions that relate the induced electric and magnetic polarization densities to the average electric and magnetic fields on the metasurface [27], [28]. The goal of the design procedure is then to find susceptibility profiles for each metasurface such that, when illuminated with the given incident field, produce a transmitted field $\vec{\Psi}^{\text {tr }}$ that closely satisfies the field specifications. Attention must also paid to ensure that the resulting susceptibility profiles can be implemented in a passive, lossless, and reciprocal way.

\section{Methodology}

This section provides a high-level overview of the procedure employed to design the cascaded metasurface pair. (More details on the implementation of the procedure will be presented in Section IV.) The goal of this procedure is to define the tangential electric and magnetic fields on either side of both

\footnotetext{
${ }^{1}$ If the desired phaseless fields are specified in the FF zone, they will be equivalent to a desired power pattern. To emphasize that the desired phase data have not been provided to the algorithm, $|\mathbf{f}|^{2}$ is used in the remaining of this paper to represent the amplitude-only specification for such cases. (|.| denotes the amplitude operator.)

${ }^{2}$ These performance criteria are first converted to a desired power pattern and are then incorporated in the inversion algorithm.
} 


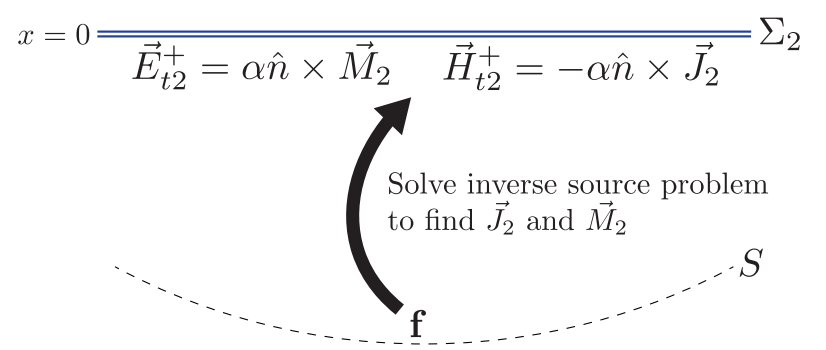

Fig. 2. First step of the cascaded metasurface design procedure. An inverse source problem is solved to find equivalent electric and magnetic currents $\vec{J}_{2}$ and $\vec{M}_{2}$ on $\Sigma_{2}^{+}$that (i) produce fields on $S$ satisfying the field specifications f, and (ii) produce null fields (Love's condition) on inward offset of $\Sigma_{2}$. Since Love's equivalence condition is enforced, the tangential output fields $\vec{E}_{t 2}^{+}$and $\vec{H}_{t 2}^{+}$can be directly computed from $\vec{J}_{2}$ and $\vec{M}_{2}$.

metasurfaces, from which the susceptibility profiles can be calculated [13]. That is, we need to determine

- tangential output fields, i.e., $\vec{E}_{t 2}^{+}$and $\vec{H}_{t 2}^{+}$on $x=0^{+}$,

- tangential input fields, i.e., $\vec{E}_{t 1}^{-}$and $\vec{H}_{t 1}^{-}$on $x=-d^{-}$, and

- tangential interior fields, i.e., $\vec{E}_{t 1}^{+}$and $\vec{H}_{t 1}^{+}$on $x=-d^{+}$, and $\vec{E}_{t 2}^{-}$and $\vec{H}_{t 2}^{-}$on $x=0^{-}$.

Noting Figure 1, in what follows, we refer to $x=0^{+}$and $x=0^{-}$as $\Sigma_{2}^{+}$and $\Sigma_{2}^{-}$respectively, and we refer to $x=-d^{+}$ and $x=-d^{-}$as $\Sigma_{1}^{+}$and $\Sigma_{1}^{-}$respectively.

\section{A. Finding the Tangential Output Fields (on $\Sigma_{2}^{+}$)}

The first step involves determining tangential electric and magnetic fields on the output side of the second (output) metasurface, denoted as $\vec{E}_{t 2}^{+}$and $\vec{H}_{t 2}^{+}$, that produce corresponding fields on $S$ that match the user-defined field specifications $\mathbf{f}$. This step is formulated as an inverse source problem as shown in Figure 2, in which the unknowns are equivalent electric and magnetic currents, $\vec{J}_{2}$ and $\vec{M}_{2}$. A cost-functional is minimized to find $\vec{J}_{2}$ and $\vec{M}_{2}$ such that these equivalent currents:

- produce electric and magnetic fields that satisfy the field specifications $\mathbf{f}$ on the region of interest $S$, and

- satisfy Love's equivalence condition (i.e., produce null fields on the input side of $\Sigma_{2}$ ).

Noting that Love's condition is enforced, the resulting equivalent currents will be related to the required tangential electric and magnetic fields as

$$
\vec{H}_{t 2}^{+}=-\alpha \hat{n} \times \vec{J}_{2} \quad \text { and } \quad \vec{E}_{t 2}^{+}=\alpha \hat{n} \times \vec{M}_{2},
$$

where $\hat{n}$ is the unit outward normal to $\Sigma_{2}$ (i.e., towards the output side) and $\alpha$ is a real-valued scaling factor. $(\hat{n}=\hat{x}$ in Figure 1.) The choice of $\alpha$ does not affect the normalized output field. As will be seen, we select $\alpha$ to ensure that total power is conserved across the cascaded metasurface structure.

\section{B. Tangential Input Fields (on $\Sigma_{1}^{-}$)}

Since the cascaded metasurface system is assumed to be reflectionless, the tangential fields on the input side of the first metasurface (i.e., at $x=-d^{-}$) are assumed to be the same as the known incident field (i.e., the tangential components of $\left.\vec{\Psi}^{\text {inc }}\right)$. Thus, $\vec{E}_{t 1}^{-}$and $\vec{H}_{t 1}^{-}$are known.

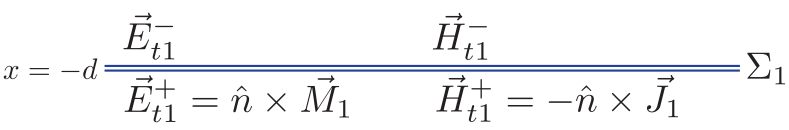

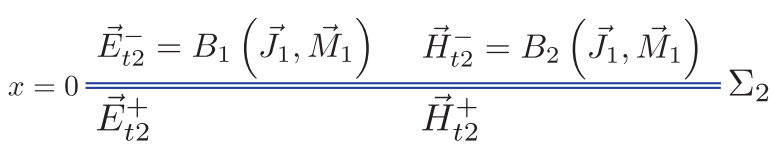

Fig. 3. Finding the interior fields in the cascaded metasurface design procedure. An inverse source problem is solved to find equivalent electric and magnetic currents $\vec{J}_{1}$ and $\vec{M}_{1}$ on $x=-d^{+}$such that (i) they result in fields satisfying local power conservation on both metasurfaces, and (ii) they produce null fields on the input side of $\Sigma_{1}$ (Love's condition). The fields $\vec{E}_{t 1}^{+}$and $\vec{H}_{t 1}^{+}$can then be directly computed from $\vec{J}_{1}$ and $\vec{M}_{1}$ since Love's condition is enforced, and $\vec{E}_{t 2}^{-}$and $\vec{H}_{t 2}^{-}$can be computed as a function of $\vec{J}_{1}$ and $\vec{M}_{1}$ through forward propagation.

\section{Finding the Tangential Interior Fields (on $\Sigma_{1}^{+}$and $\Sigma_{2}^{-}$)}

The next step is to determine the tangential electric and magnetic fields in the interior region between the two metasurfaces. More specifically, we want to determine $\vec{E}_{t 1}^{+}, \vec{H}_{t 1}^{+}$, $\vec{E}_{t 2}^{-}$, and $\vec{H}_{t 2}^{-}$. This is accomplished by formulating and solving a second inverse source problem, as shown in Figure 3. In this step, the goal is to find equivalent currents $\vec{J}_{1}$ and $\vec{M}_{1}$ on $\Sigma_{1}^{+}$ such that

- their corresponding fields satisfy LPC on both metasurfaces, and

- they satisfy Love's condition (i.e., produce null fields on the input side of $\Sigma_{1}$ ).

On $\Sigma_{1}$, satisfying LPC means that the time-averaged real power density in the $\hat{x}$ direction of $\vec{E}_{t 1}^{+}$and $\vec{H}_{t 1}^{+}$must be locally (i.e., at each unit cell) equal to the real power density of $\vec{E}_{t 1}^{-}$and $\vec{H}_{t 1}^{-}$in the $\hat{x}$ direction. As in Section III-A, since Love's condition is enforced the necessary fields are related to the equivalent currents as

$$
\vec{H}_{t 1}^{+}=-\hat{n} \times \vec{J}_{1} \quad \text { and } \quad \vec{E}_{t 1}^{+}=\hat{n} \times \vec{M}_{1} .
$$

On $\Sigma_{2}$, the LPC condition is between the previously defined output fields, $\vec{E}_{t 2}^{+}$and $\vec{H}_{t 2}^{+}$, (Section III-A) and the fields $\vec{E}_{t 2}^{-}$ and $\vec{H}_{t 2}^{-}$that are computed from the equivalent currents using

$$
\vec{E}_{t 2}^{-}=B_{1}\left(\vec{J}_{1}, \vec{M}_{1}\right) \quad \text { and } \quad \vec{H}_{t 2}^{-}=B_{2}\left(\vec{J}_{1}, \vec{M}_{1}\right)
$$

where $B_{1}$ and $B_{2}$ are electric and magnetic field integral equation operators, respectively.

\section{Computing the Susceptibility Profiles}

Once the tangential fields on either side of both metasurfaces have been found, we can apply the generalized sheet transition conditions (GSTCs) [27], [28] to compute the surface susceptibility profiles required to support the two intended field discontinuities. Assuming a planar metasurface on the $z y$ plane for convenience, adopting a time-dependency of $e^{j \omega t}$, and neglecting the normal component of the polarization 
densities for mathematical simplicity, the GSTCs (at a given location on the metasurface) can be simplified to

$$
\begin{aligned}
\left(\begin{array}{c}
-\Delta H_{z} \\
\Delta H_{y}
\end{array}\right)=j \omega \epsilon_{0}\left(\begin{array}{ll}
\chi_{\mathrm{ee}}^{y y} & \chi_{\mathrm{ee}}^{y z} \\
\chi_{\mathrm{ee}}^{z y} & \chi_{\mathrm{ee}}^{z z}
\end{array}\right)\left(\begin{array}{l}
E_{y, \mathrm{av}} \\
E_{z, \mathrm{av}}
\end{array}\right) \\
+j \omega \sqrt{\epsilon_{0} \mu_{0}}\left(\begin{array}{ll}
\chi_{\mathrm{em}}^{y y} & \chi_{\mathrm{em}}^{y z} \\
\chi_{\mathrm{em}}^{z y} & \chi_{\mathrm{em}}^{z z}
\end{array}\right)\left(\begin{array}{l}
H_{y, \mathrm{av}} \\
H_{z, \mathrm{av}}
\end{array}\right) \\
\left(\begin{array}{c}
-\Delta E_{y} \\
\Delta E_{z}
\end{array}\right)=j \omega \mu_{0}\left(\begin{array}{ll}
\chi_{\mathrm{mm}}^{z z} & \chi_{\mathrm{mm}}^{z y} \\
\chi_{\mathrm{mm}}^{y y} & \chi_{\mathrm{mm}}^{y y}
\end{array}\right)\left(\begin{array}{l}
H_{z, \mathrm{av}} \\
H_{y, \mathrm{av}}
\end{array}\right) \\
+j \omega \sqrt{\epsilon_{0} \mu_{0}}\left(\begin{array}{ll}
\chi_{\mathrm{me}}^{z z} & \chi_{\mathrm{me}}^{z y} \\
\chi_{\mathrm{me}}^{y z} & \chi_{\mathrm{me}}^{y y}
\end{array}\right)\left(\begin{array}{l}
E_{z, \mathrm{av}} \\
E_{y, \mathrm{av}}
\end{array}\right)
\end{aligned}
$$

where $\epsilon_{0}$ and $\mu_{0}$ are the permittivity and permeability of free space and $\omega$ is the angular frequency of the timeharmonic fields. The surface susceptibility terms $\chi$ represent the electric/magnetic response (denoted by the first subscript) to an applied electric/magnetic field (denoted by the second subscript). For a metasurface $\Sigma_{i}$ (where $i=1,2$ ), the average and difference fields in (4) are defined as

$$
\begin{aligned}
& \vec{\Psi}_{\mathrm{av}} \triangleq \frac{\vec{\Psi}^{+}+\vec{\Psi}^{-}}{2}, \\
& \Delta \vec{\Psi} \triangleq \vec{\Psi}^{+}-\vec{\Psi}^{-} .
\end{aligned}
$$

Under certain assumptions that are discussed in Section IV-H, (4) can be reduced to a well-defined system of equations that allows for (unique) analytic solutions for the remaining susceptibility terms for a given field transformation.

\section{E. Conversion to Three-Layer Admittance Model}

Once the surface susceptibility profiles are determined, they can be converted to three-layer admittance-sheet models given a known dielectric substrate (e.g., Rogers RO3010). This can then be simulated (in our case, in ANSYS HFSS) to verify the performance of the cascaded metasurface system with respect to the desired specifications.

\section{NUMERICAL IMPLEMENTATION OF THE INVERSION FRAMEWORK}

The following numerical implementation assumes onedimensional (1D) metasurfaces along $y$ with $\mathrm{TE}_{z}$ fields propagating in the $x y$ plane, although the framework can be applied to other geometries and field polarizations. (A $\mathrm{TM}_{z}$ example will be considered in Section V-C.)

\section{A. Forming the Vectors of Unknowns}

Each of the two metasurfaces is discretized into $N$ unit cells (although the number of unit cells on each metasurface do not necessarily need to be equal). The discrete vector of unknown electric and magnetic equivalent currents on $\Sigma_{1}^{+}$(i.e., on $x=-d^{+}$) are denoted as $\mathbf{J}_{1}$ and $\mathbf{M}_{1}$, which are discretized using pulse basis functions. These currents can be written in terms of their real and imaginary components as

$$
\begin{aligned}
\mathbf{J}_{1} & =\mathbf{J}_{1, \mathrm{R}}+j \mathbf{J}_{1, \mathrm{I}}, \\
\mathbf{M}_{1} & =\mathbf{M}_{1, \mathrm{R}}+j \mathbf{M}_{1, \mathrm{I}} .
\end{aligned}
$$

We then concatenate the real and imaginary parts of the equivalent currents into a single vector as

$$
\mathbf{x}_{1}=\left[\begin{array}{c}
\mathbf{J}_{1, \mathrm{R}} \\
\mathbf{J}_{1, \mathrm{I}} \\
\mathbf{M}_{1, \mathrm{R}} \\
\mathbf{M}_{1, \mathrm{I}}
\end{array}\right]
$$

With this organization, we will perform the optimization over the real-valued vector $\mathbf{x}_{1} \in \mathbb{R}^{4 N}$ as opposed to the complex equivalent currents $\mathbf{J}_{\mathbf{1}} \in \mathbb{C}^{N}$ and $\mathbf{M}_{1} \in \mathbb{C}^{N}$.

Similarly, we perform the same re-structuring of the equivalent currents $\mathbf{J}_{2}$ and $\mathbf{M}_{2}$ on $\Sigma_{2}^{+}$(i.e., on $x=0^{+}$) to construct the second vector of unknowns denoted by $\mathbf{x}_{2}$. Assuming the same number of unit cells for the second metasurface, then $\mathbf{x}_{2} \in \mathbb{R}^{4 N}$. Thus, we now have two (real-valued) vectors of unknowns: $\mathbf{x}_{1}$ and $\mathbf{x}_{2}$. As described in Section III, we first need to determine $\mathbf{x}_{2}$, and then we will find $\mathbf{x}_{1}$.

\section{B. Forming a Data Misfit Cost Functional with Respect to $\mathbf{x}_{2}$}

The first step involves forming a cost functional with respect to $\mathbf{x}_{2}$ representing the discrepancy between the field specifications $\mathbf{f}$ on $S$ and those generated by a predicted $\mathbf{x}_{2}$, as described in Section III-A. Let us assume that the desired specifications are amplitude-only fields on $S$. That is, let the field specifications be $|\mathbf{f}|^{2}$, a vector of squared field amplitude at the discrete points of $S .^{3}$ The data misfit cost functional associated with these specifications is a mapping from $\mathbb{R}^{4 N}$ to $\mathbb{R}$, and is expressed as

$$
\mathcal{C}_{\mathrm{F}}\left(\mathbf{x}_{2}\right)=\frac{\left\|\left|\mathbf{A}_{\mathrm{F}} \mathbf{x}_{2}\right|^{2}-|\mathbf{f}|^{2}\right\|_{S}^{2}}{\left\||\mathbf{f}|^{2}\right\|_{S}^{2}}
$$

where $\mathbf{A}_{\mathrm{F}}$ is the discretized integral operator that produces $H_{z}$ (due to $\mathrm{TE}_{z}$ assumption) at the locations of the field specifications in $|\mathbf{f}|^{2}$ from the equivalent currents $\mathrm{x}_{2} \cdot{ }^{4}$ In addition, $\|\cdot\|_{S}$ denotes the $L^{2}$-norm over $S$. Later on in Section V-B, we will also consider a case where the field specifications are instead FF performance criteria such as main beam directions, HPBW, and null locations. In such cases, these performance criteria need to be first converted to a desired power pattern $|\mathbf{f}|^{2}$. The procedure to perform this conversion can be found in [17].

In addition, we note that Love's condition is enforced by adding 'virtual' data to $|\mathbf{f}|^{2}$. This is done by augmenting $|\mathbf{f}|^{2}$ with a vector of zeros associated with the locations at which the null field is desired [17]..$^{5}$ In this case, these nulls are enforced on a line which is an inward offset of $\Sigma_{2}^{+}$. Similarly, the matrix $\mathbf{A}_{\mathrm{F}}$ needs to be augmented such that when it operates on $\mathbf{x}_{2}$, the operation results in the fields produced by $\mathrm{x}_{2}$ in the region of interest $S$ and in the domain at which null fields are desired.

\footnotetext{
${ }^{3}$ If $S$ is in the FF zone, $|\mathbf{f}|^{2}$ is equivalent to a desired power pattern.

${ }^{4}$ For $\mathrm{TM}_{z}$ polarization the operator $\mathbf{A}_{\mathrm{F}}$ would produce $E_{z}$ at the locations of interest.

${ }^{5}$ This method to enforce Love's condition is based on enforcing Love's condition for the antenna diagnostic problem [29], and has also been applied to the inverse source problem associated with noninvasive specific absorption rate characterization [30].
} 


\section{Forming a Total Variation Regularizer for $\mathbf{x}_{2}$}

We now introduce an additive regularization term based on the $L^{2}$-norm total variation (TV) regularizer. ${ }^{6}$ This functional, denoted as $\mathcal{C}_{\mathrm{TV}}\left(\mathbf{x}_{2}\right)$, penalizes solutions with rapid variations from cell to cell and is given by

$$
\mathcal{C}_{\mathrm{TV}}\left(\mathbf{x}_{2}\right)=w_{\mathrm{TV}}\left\|D_{y}\left(\mathbf{x}_{2}\right)\right\|_{\Sigma_{2}}^{2}
$$

where $w_{\mathrm{TV}}$ is a real-valued weighting parameter. The operator $D_{y}\left(\mathbf{x}_{2}\right)$ applies a derivative with respect to $y$ to the individual current components of $\mathbf{x}_{2}$ separately, explicitly stated as

$$
D_{y}\left(\mathbf{x}_{2}\right) \triangleq\left[\begin{array}{c}
\frac{\partial}{\partial y} \mathbf{J}_{2, \mathrm{R}} \\
\frac{\partial}{\partial y} \mathbf{J}_{2, \mathrm{I}} \\
\frac{\partial}{\partial y} \mathbf{M}_{2, \mathrm{R}} \\
\frac{\partial}{\partial y} \mathbf{M}_{2, \mathrm{I}}
\end{array}\right] .
$$

This regularization term biases the solution towards a higher degree of continuity in the equivalent currents (and therefore fields) from unit cell to unit cell. This is especially important because in Section IV-F we attempt to enforce LPC at each unit cell on $\Sigma_{2}$, and rapidly varying output fields could make this more difficult or even practically impossible given the finite size of the metasurfaces. A secondary benefit of this regularization is apparent when considering microscopic metasurface design, i.e., physical unit cell design. The typical procedure is to design and simulate each unit cell independently while assuming infinite periodicity. When each unit cell is then placed in the final metasurface structure, the periodicity assumption no longer holds and the behaviour of each unit cell will deviate from what was expected. The inclusion of the TV regularizer reduces the variation from cell to cell, thereby reducing the error in the periodicity assumption.

\section{Iterative Minimization to Determine $\mathbf{x}_{2}$}

Combining (9) and (10) results in the total cost functional (or, regularized cost functional) that is minimized during the first optimization step, explicitly written as

$$
\mathcal{C}_{2}\left(\mathbf{x}_{2}\right)=\underbrace{\mathcal{C}_{\mathrm{F}}\left(\mathbf{x}_{2}\right)}_{\text {data misfit }}+\underbrace{\mathcal{C}_{\mathrm{TV}}\left(\mathbf{x}_{2}\right)}_{\text {smoothness }} .
$$

Minimization is performed in an iterative fashion using the nonlinear conjugate gradient (CG) method. At the $k^{\text {th }}$ iteration, the solution update is

$$
\mathbf{x}_{2, k+1}=\mathbf{x}_{2, k}-\beta_{k} \mathbf{v}_{k}
$$

where $\beta_{k}$ is the real-valued step length. The vector $\mathbf{v}_{k}$ is the CG direction at the $k^{\text {th }}$ CG iteration computed using PolakRibière formula [33]

$$
\mathbf{v}_{k}=\mathbf{g}_{2, k}+\frac{\left(\mathbf{g}_{2, k}-\mathbf{g}_{2, k-1}\right)^{\mathrm{H}} \mathbf{g}_{2, k}}{\mathbf{g}_{2, k-1}^{\mathrm{H}} \mathbf{g}_{2, k-1}} \mathbf{v}_{k-1}
$$

where the superscript " $\mathrm{H}$ " denotes the Hermitian operation (complex conjugate transpose) and $\mathbf{g}_{2, k}$ and $\mathbf{g}_{2, k-1}$ are the

\footnotetext{
${ }^{6}$ The TV regularizer has been utilized in microwave imaging [25], [26] NF antenna measurements and characterization [31] as well as image deblurring [32]. One of the reasons behind the use of this regularizer has been its smoothing effect on spurious oscillations in the reconstructed solution.
}

gradients of (12) with respect to $\mathbf{x}_{2}$ at iterations $k$ and $k-1$, respectively. As derived in Appendix A, the gradient of (12) consists of a sum of the gradients of (9) and (10), the first of which is

$$
\mathbf{g}_{\mathrm{F}}\left(\mathbf{x}_{2}\right)=\frac{4 \operatorname{Re}\left\{\mathbf{A}_{\mathrm{F}}^{\mathrm{H}}\left(\mathbf{r}_{\mathrm{F}} \odot \mathbf{A}_{\mathrm{F}} \mathbf{x}_{2}\right)\right\}}{\left\||\mathbf{f}|^{2}\right\|_{S}^{2}}
$$

where " $\odot$ " indicates a Hadamard (element-wise) product of two vectors, 'Re' denotes the real-part operator ${ }^{7}$, and $\mathbf{r}_{\mathrm{F}}$ is the residual vector of (9) defined as

$$
\mathbf{r}_{\mathrm{F}}=\left|\mathbf{A}_{\mathrm{F}} \mathbf{x}_{2}\right|^{2}-|\mathbf{f}|^{2} \text {. }
$$

The gradient of (10) is

$$
\mathbf{g}_{\mathrm{TV}}\left(\mathbf{x}_{2}\right)=-2 w_{\mathrm{TV}} D_{y}^{2}\left(\mathbf{x}_{2}\right)
$$

where the operator $D_{y}^{2}\left(\mathbf{x}_{2}\right)$, similarly to (11), computes the second derivative (Laplacian operator) with respect to $y$ of the individual current components of $\mathbf{x}_{2}$ as

$$
D_{y}^{2}\left(\mathbf{x}_{2}\right)=\left[\begin{array}{c}
\frac{\partial^{2}}{\partial y^{2}} \mathbf{J}_{2, \mathrm{R}} \\
\frac{\partial^{2}}{\partial y^{2}} \mathbf{J}_{2, \mathrm{I}} \\
\frac{\partial^{2}}{\partial y^{2}} \mathbf{M}_{2, \mathrm{R}} \\
\frac{\partial^{2}}{\partial y^{2}} \mathbf{M}_{2, \mathrm{I}}
\end{array}\right] .
$$

Both (11) and (18) are evaluated numerically using a central difference approximation. The gradient required to update the search direction $\mathbf{v}_{k}$ in (14) can then be computed as

$$
\mathbf{g}_{2, k}=\mathbf{g}_{\mathrm{F}, k}+\mathbf{g}_{\mathrm{TV}, k} .
$$

The iterative procedure is terminated when the change in $\mathrm{x}_{2}$ from one CG iteration to the next drops below a preset tolerance, typically $10^{-6 \%}$.

\section{E. Enforcing Total Power Conservation to Find $\vec{E}_{t 2}^{+}$and $\vec{H}_{t 2}^{+}$}

Upon convergence, the above iterative minimization will yield an appropriate $\mathbf{x}_{2}$. Subsequently, we need to compute the corresponding output tangential fields on $\Sigma_{2}^{+}$using (1). However, we first need to compute the scaling factor $\alpha$ needed to ensure that total power is conserved across the cascaded metasurface structure. (Note that this is not related to LPC.) To do so, we define $\mathbf{p}_{\text {in }} \in \mathbb{R}^{N}$ as the vector containing the timeaverage real power density of $\vec{E}_{t 1}^{-}$and $\vec{H}_{t 1}^{-}$normally incident on $\Sigma_{1}^{-}$, with the $i^{\text {th }}$ element of $\mathbf{p}_{\text {in }}$ defined as

$$
p_{\text {in }, i}=\left.\frac{1}{2} \operatorname{Re}\left(\vec{E}_{t 1}^{-} \times \vec{H}_{t 1}^{-*}\right)\right|_{\text {unit cell } i} .
$$

In this work, as noted in Section III-B, we are concerned with the design of reflectionless metasurfaces, and thus $\mathbf{p}_{\text {in }}$ can be calculated with knowledge of the incident field only. Thus, the total incident power will be the summation of the elements of the vector $\mathbf{p}_{\text {in }}$, denoted by $\operatorname{sum}\left(\mathbf{p}_{\text {in }}\right)$.

\footnotetext{
${ }^{7}$ Note that since $\mathbf{x}_{2}$ is a real-valued vector, the gradient vector is naturally a real-valued vector as well.
} 
The time-average real power density of the output field can be written in terms of the equivalent currents as [18]

$$
\mathbf{p}_{\text {out }}=\frac{\alpha^{2}}{2}\left(\mathbf{J}_{2, \mathrm{R}} \odot \mathbf{M}_{2, \mathrm{R}}+\mathbf{J}_{2, \mathrm{I}} \odot \mathbf{M}_{2, \mathrm{I}}\right) .
$$

Note that since we have already determined $\mathbf{x}_{2}$ in the previous step, we also know $\mathbf{J}_{2, \mathrm{R}}, \mathbf{J}_{2, \mathrm{I}}, \mathbf{M}_{2, \mathrm{R}}$, and $\mathbf{M}_{2, \mathrm{I}}$ based on the concatenation convention used in Section IV-A. If we now select $\alpha$ to be

$$
\alpha=\left(\frac{\operatorname{sum}\left(\mathbf{p}_{\text {in }}\right)}{\frac{1}{2} \operatorname{sum}\left(\mathbf{J}_{2, \mathrm{R}} \odot \mathbf{M}_{2, \mathrm{R}}+\mathbf{J}_{2, \mathrm{I}} \odot \mathbf{M}_{2, \mathrm{I}}\right)}\right)^{\frac{1}{2}}
$$

the total output power will be equal to the total input power, i.e., $\operatorname{sum}\left(\mathbf{p}_{\text {out }}\right)=\operatorname{sum}\left(\mathbf{p}_{\text {in }}\right)$, thus satisfying TPC. Finally, having found $\alpha, \mathbf{J}_{2, \mathrm{R}}, \mathbf{J}_{2, \mathrm{I}}, \mathbf{M}_{2, \mathrm{R}}$, and $\mathbf{M}_{2, \mathrm{I}}$, we can now use (1) to determine the required output tangential fields $\vec{E}_{t 2}^{+}$and $\vec{H}_{t 2}^{+}$on $\Sigma_{2}^{+}$.

\section{F. Forming the Cost Functional with Respect to $\mathbf{x}_{1}$}

The goal of this step is to determine the fields between the two metasurfaces that result in the intended field transformation while satisfying LPC on both metasurfaces. This involves finding a set of equivalent electric and magnetic currents $\mathbf{x}_{1}$ on $\Sigma_{1}^{+}$, as defined in (8). The cost functional that is minimized in this step is a mapping from $\mathbb{R}^{4 N}$ to $\mathbb{R}$, and consists of the following four terms

$$
\mathcal{C}_{1}\left(\mathbf{x}_{1}\right)=\underbrace{\mathcal{C}_{\mathrm{L}}\left(\mathbf{x}_{1}\right)}_{\text {Love's cond. }}+\underbrace{\mathcal{C}_{\mathrm{P}, 1}\left(\mathbf{x}_{1}\right)}_{\text {LPC on } \Sigma_{1}}+\underbrace{\mathcal{C}_{\mathrm{P}, 2}\left(\mathbf{x}_{1}\right)}_{\text {LPC on } \Sigma_{2}}+\underbrace{\mathcal{C}_{\mathrm{TV}}\left(\mathbf{x}_{1}\right)}_{\text {smoothness }} .
$$

The first term is defined as

$$
\mathcal{C}_{\mathrm{L}}\left(\mathbf{x}_{1}\right)=\left\|\mathbf{L} \mathbf{x}_{1}\right\|_{\Sigma_{1}}^{2}
$$

where $\mathbf{L}$ is a discretized integral operator that produces $H_{z}$ (or $E_{z}$ for $\mathrm{TM}_{z}$ polarization) at the locations where Love's condition is enforced for $\Sigma_{1}$. Minimizing $\mathcal{C}_{\mathrm{L}}\left(\mathbf{x}_{1}\right)$ ensures that $\mathbf{x}_{1}$ produces null fields on the $x=-d^{-}$side of $\Sigma_{1}$ and allows us to eventually calculate the tangential fields using (2).

The second term in (23) is used to enforce LPC on $\Sigma_{1}$ as originally derived in [18], defined as

$$
\mathcal{C}_{\mathrm{P}, 1}\left(\mathbf{x}_{1}\right)=\frac{w_{1}\left\|\frac{1}{2}\left(\mathbf{J}_{1, \mathrm{R}} \odot \mathbf{M}_{1, \mathrm{R}}+\mathbf{J}_{1, \mathrm{I}} \odot \mathbf{M}_{1, \mathrm{I}}\right)-\mathbf{p}_{\text {in }}\right\|_{\Sigma_{1}}^{2}}{\left\|\mathbf{p}_{\text {in }}\right\|_{\Sigma_{1}}^{2}}
$$

which quantifies the difference between the real power density of $\vec{E}_{t 1}^{+}$and $\vec{H}_{t 1}^{+}$and the known incident power $\mathbf{p}_{\text {in }}$ in the $x$ direction at each unit cell on $\Sigma_{1}$. The real-valued weighting parameter $w_{1}$ is included to balance the contribution of $\mathcal{C}_{\mathrm{P}, 1}\left(\mathbf{x}_{1}\right)$ to the overall functional. As noted earlier, enforcing LPC is necessary to ensure that the resulting metasurface can be implemented in a passive and lossless manner [20].

The third term in (23) is used to enforce LPC on $\Sigma_{2}$. In terms of the tangential fields, this condition can be explicitly written as

$$
\left.\frac{1}{2} \operatorname{Re}\left(\vec{E}_{t 2}^{-} \times \vec{H}_{t 2}^{-*}\right)\right|_{\text {unit cell } i}=\left.\underbrace{\frac{1}{2} \operatorname{Re}\left(\vec{E}_{t 2}^{+} \times \vec{H}_{t 2}^{+*}\right)}_{\text {known from Section IV-E }}\right|_{\text {unit cell } i}
$$

which must hold at each unit cell on $\Sigma_{2}$. Analogously to (20), we evaluate the right-hand side of (26) for each unit cell to generate the vector $\mathbf{p}_{\text {out }}$ containing the local real power densities of the fields $\vec{E}_{t 2}^{+}$and $\vec{H}_{t 2}^{+}$. We also define discretized integral operators $\mathbf{B}_{1}$ and $\mathbf{B}_{\mathbf{2}}$ corresponding to (3), which respectively produce the tangential electric and magnetic fields at each unit cell on $\Sigma_{2}^{-}$from the equivalent currents $\mathbf{x}_{1}$. We can then write $\mathcal{C}_{\mathrm{P}, 2}\left(\mathbf{x}_{1}\right)$ as

$$
\mathcal{C}_{\mathrm{P}, 2}\left(\mathbf{x}_{1}\right)=\frac{w_{2}\left\|\frac{1}{2} \operatorname{Re}\left(\mathbf{B}_{1} \mathbf{x}_{1} \odot \mathbf{B}_{2}^{*} \mathbf{x}_{1}\right)-\mathbf{p}_{\text {out }}\right\|_{\Sigma_{2}}^{2}}{\left\|\mathbf{p}_{\text {out }}\right\|_{\Sigma_{2}}^{2}}
$$

where $w_{2}$ is a real-valued weighting parameter. The final term of (23), $\mathcal{C}_{\mathrm{TV}}\left(\mathbf{x}_{1}\right)$, performs the same operation previously defined in (10) (but operating on $\mathbf{x}_{1}$ instead of $\mathbf{x}_{2}$ ).

\section{G. Iterative Minimization to Determine $\mathrm{x}_{1}$}

As in Section IV-D, the cost functional in (23) is minimized using the nonlinear CG method with update equations similar to (13) and (14). As derived in Appendix B, the gradient of $\mathcal{C}_{\mathrm{L}}\left(\mathrm{x}_{1}\right)$ is

$$
\mathbf{g}_{\mathbf{L}}\left(\mathbf{x}_{1}\right)=2 \operatorname{Re}\left(\mathbf{L}^{\mathrm{H}} \mathbf{L} \mathbf{x}_{1}\right)
$$

and the gradient of $\mathcal{C}_{\mathrm{P}, 1}\left(\mathbf{x}_{1}\right)$ is

$$
\mathbf{g}_{\mathrm{P}, 1}\left(\mathbf{x}_{1}\right)=\frac{w_{1}}{\left\|\mathbf{p}_{\text {in }}\right\|_{\Sigma_{1}}^{2}}\left[\begin{array}{c}
\mathbf{r}_{1} \odot \mathbf{M}_{1, \mathrm{R}} \\
\mathbf{r}_{1} \odot \mathbf{M}_{1, \mathrm{I}} \\
\mathbf{r}_{1} \odot \mathbf{J}_{1, \mathrm{R}} \\
\mathbf{r}_{1} \odot \mathbf{J}_{1, \mathrm{I}}
\end{array}\right]
$$

where the residual vector $\mathbf{r}_{1}$ is defined as

$$
\mathbf{r}_{1}\left(\mathbf{x}_{1}\right)=\frac{1}{2}\left(\mathbf{J}_{1, \mathrm{R}} \odot \mathbf{M}_{1, \mathrm{R}}+\mathbf{J}_{1, \mathrm{I}} \odot \mathbf{M}_{1, \mathrm{I}}\right)-\mathbf{p}_{\text {in }} .
$$

In addition, the gradient of $\mathcal{C}_{\mathrm{P}, 2}\left(\mathbf{x}_{1}\right)$ is

$$
\mathbf{g}_{\mathrm{P}, 2}\left(\mathbf{x}_{1}\right)=\frac{w_{2} \operatorname{Re}\left[\operatorname{diag}\left(\mathbf{B}_{1} \mathbf{x}_{1}\right) \mathbf{B}_{2}^{*}+\operatorname{diag}\left(\mathbf{B}_{2}^{*} \mathbf{x}_{1}\right) \mathbf{B}_{1}\right]^{\mathrm{T}} \mathbf{r}_{2}}{\left\|\mathbf{p}_{\text {out }}\right\|_{\Sigma_{2}}^{2}}
$$

where the ' $\operatorname{diag}(\cdot)$ ' operator generates a diagonal matrix from a vector and the superscript ' $\mathrm{T}$ ' denotes the transpose operator. The residual vector $\mathbf{r}_{2}$ is defined as

$$
\mathbf{r}_{2}\left(\mathbf{x}_{1}\right)=\frac{1}{2} \operatorname{Re}\left(\mathbf{B}_{1} \mathbf{x}_{1} \odot \mathbf{B}_{2}^{*} \mathbf{x}_{1}\right)-\mathbf{p}_{\text {out }} .
$$

The gradient of $\mathcal{C}_{\mathrm{TV}}\left(\mathbf{x}_{1}\right)$ is computed in the same way as (17) but now operates on $\mathbf{x}_{1}$. In addition, the iterative procedure is terminated in the same way as in Section IV-D, when the solution stagnates as determined by a preset tolerance.

Finally, once we have a solution for $\mathbf{x}_{1}$, we use (2) to compute the tangential fields $\vec{E}_{t 1}^{+}$and $\vec{H}_{t 1}^{+}$. We then use (3) to compute the tangential fields $\vec{E}_{t 2}^{-}$and $\vec{H}_{t 2}^{-}$. Let us also remind ourselves that we have already determined $\vec{E}_{t 2}^{+}$and $\vec{H}_{t 2}^{+}$in Section IV-E, and we already know $\vec{E}_{t 1}^{-}$and $\vec{H}_{t 1}^{-}$from Section III-B. Therefore, we now know the tangential fields on both metasurfaces, and can therefore determine the required surface susceptibility profiles for both of these metasurfaces. Note that since we have enforced LPC for each individual metasurface, we can implement the desired transformation using lossless and passive metasurfaces. 


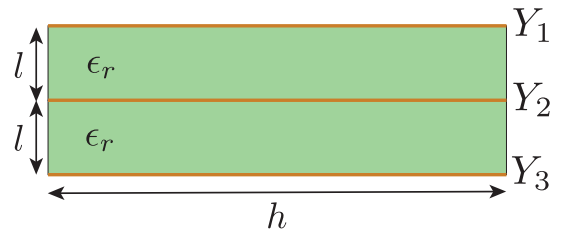

Fig. 4. The three-layer admittance sheet topology used for each unit cell [35] Two substrate layers with relative permittivity $\epsilon_{r}$ and thickness $l$ are placed between three admittance sheets $Y_{1}, Y_{2}$, and $Y_{3}$. Also, $h$ represents the size of the unit cell. (The bondply layer has not been included in this model.) From a circuit viewpoint, this can be seen as five cascaded two-port networks: three shunt admittances $\left(Y_{1}, Y_{2}\right.$, and $\left.Y_{3}\right)$ and two transmission line sections of length $l[14]$.

\section{H. Susceptibility Profile Calculation}

Once the tangential fields have been determined on both sides of $\Sigma_{1}$ and $\Sigma_{2}$ we can apply the GSTCs to determine the required susceptibility profiles to support the two field discontinuities. For 1D metasurfaces along $y$ with $\mathrm{TE}_{z}$ fields, (4) at a given unit cell simplifies to ${ }^{8}$

$$
\begin{aligned}
& -\Delta H_{z}=\left(j \omega \epsilon_{0} E_{y, \mathrm{av}}\right) \chi_{\mathrm{ee}}^{y y}+\left(j \omega \sqrt{\mu_{0} \epsilon_{0}} H_{z, \mathrm{av}}\right) \chi_{\mathrm{em}}^{y z} \\
& -\Delta E_{y}=\left(j \omega \mu_{0} H_{z, \mathrm{av}}\right) \chi_{\mathrm{mm}}^{z z}+\left(j \omega \sqrt{\mu_{0} \epsilon_{0}} E_{y, \mathrm{av}}\right) \chi_{\mathrm{me}}^{z y}
\end{aligned}
$$

In order to ensure that the metasurfaces are passive, lossless, and reciprocal, we follow the procedure in [18] which stipulates that $\chi_{\mathrm{ee}}^{y y}$ and $\chi_{\mathrm{mm}}^{z z}$ are purely real $(\mathbb{R})$ while $\chi_{\mathrm{em}}^{y z}$ and $\chi_{\text {me }}^{z y}$ are purely imaginary (I). This restriction allows (33) to be solved analytically on each metasurface, with a unique solution for each unit cell. ${ }^{9}$ (Any field transformation that satisfies LPC can be supported in this manner due to the inclusion of magnetoelectric coupling [19].) Note that since (i) we have already satisfied LPC in Section IV-F, and (ii) $\chi_{\text {ee }}^{y y} \in \mathbb{R}$ and $\chi_{\mathrm{mm}}^{z z} \in \mathbb{R}$ cannot create any loss, this will inherently result in the losslessness condition $\chi_{\mathrm{me}}^{z y}=\left(\chi_{\mathrm{em}}^{y z}\right)^{*}$ [34, Appendix B] being satisfied. Finally, the stipulation $\chi_{\mathrm{me}}^{z y} \in \mathbb{I}$ and $\chi_{\mathrm{em}}^{y z} \in \mathbb{I}$ causes the relation $\chi_{\mathrm{me}}^{z y}=\left(\chi_{\mathrm{em}}^{y z}\right)^{*}$ to be equivalent to $\chi_{\mathrm{me}}^{z y}=$ $-\chi_{\mathrm{em}}^{y z}$, which is the condition for reciprocity [34, Sec. 2.2], and therefore the resulting metasurface is also reciprocal. Thus, in the next section, we have substituted $\chi_{\mathrm{me}}^{z y}$ with $-\chi_{\mathrm{em}}^{y z}$.

\section{Conversion to the Three-Layer Model}

In order to simulate the metasurfaces using commercial software, we employ a three-layer admittance sheet topology [23], [35]-[37] for each unit cell as shown in Figure 4. The conversion from the susceptibility profiles to the admittance sheet model is performed using the procedure in [38]. At a given unit cell, we start by rearranging (33) into the ABCD representation of a two-port network as

$$
\left[\begin{array}{c}
\vec{E}_{t i}^{-} \\
\vec{H}_{t i}^{-}
\end{array}\right]=\left[\begin{array}{ll}
A & B \\
C & D
\end{array}\right]\left[\begin{array}{l}
\vec{E}_{t i}^{+} \\
\vec{H}_{t i}^{+}
\end{array}\right],
$$

\footnotetext{
${ }^{8}$ Later on, in Section V-C, we consider a $\mathrm{TM}_{z}$ case as well.

${ }^{9}$ Note that (33) represents two complex equations which are equivalent to four real equations. On the other hand, since $\chi_{\mathrm{ee}}^{y y} \in \mathbb{R}, \chi_{\mathrm{mm}}^{z z} \in \mathbb{R}, \chi_{\mathrm{em}}^{y z} \in \mathbb{I}$ and $\chi_{\mathrm{me}}^{z y} \in \mathbb{I}$ we essentially have four real unknowns as well; thus, this system of equations has a unique solution.
}

where $i=1,2$ for the first and second metasurfaces respectively. This results in the following ABCD parameters in terms of the surface susceptibility values

$$
\begin{aligned}
& A=G^{-1}\left[\frac{k_{0}^{2}}{4} \chi_{\mathrm{ee}}^{y y} \chi_{\mathrm{mm}}^{z z}-\left(1-\frac{j k_{0}}{2} \chi_{\mathrm{em}}^{y z}\right)^{2}\right] \\
& B=G^{-1}\left[-j \omega \mu_{0} \chi_{\mathrm{mm}}^{z z}\right] \\
& C=G^{-1}\left[-j \omega \epsilon_{0} \chi_{\mathrm{ee}}^{y y}\right] \\
& D=G^{-1}\left[\frac{k_{0}^{2}}{4} \chi_{\mathrm{ee}}^{y y} \chi_{\mathrm{mm}}^{z z}-\left(1+\frac{j k_{0}}{2} \chi_{\mathrm{em}}^{y z}\right)^{2}\right]
\end{aligned}
$$

where $G$ is

$$
G=-\left(\frac{k_{0}}{2} \chi_{\mathrm{em}}^{y z}\right)^{2}-\frac{k_{0}^{2}}{4} \chi_{\mathrm{ee}}^{y y} \chi_{\mathrm{mm}}^{z z}-1 .
$$

It is worthwhile to note that $A D-B C=1$ (the criterion for a reciprocal network). In addition, note that since $\chi_{\mathrm{ee}}^{y y} \in \mathbb{R}$, $\chi_{\mathrm{mm}}^{z z} \in \mathbb{R}$ and $\chi_{\mathrm{em}}^{y z} \in \mathbb{I}$, then we will have $A \in \mathbb{R}, D \in \mathbb{R}$, $B \in \mathbb{I}$, and $C \in \mathbb{I}$, which further indicates a lossless two-port network.

The $A B C D$ parameters of the three-layer admittance sheet topology can be computed using transmission line theory [35] and equated with (35), allowing the three admittance sheet values $Y_{1}, Y_{2}$, and $Y_{3}$ to be calculated as ${ }^{10}$

$$
\begin{aligned}
Y_{1} & =\frac{D-j Z_{0} \sin (\beta l) \cos (\beta l) Y_{2}-\cos ^{2}(\beta l)+\sin ^{2}(\beta l)}{2 j Z_{0} \sin (\beta l) \cos (\beta l)-Z_{0}^{2} \sin ^{2}(\beta l) Y_{2}} \\
Y_{2} & =\frac{B-2 j Z_{0} \sin (\beta l) \cos (\beta l)}{-Z_{0}^{2} \sin ^{2}(\beta l)} \\
Y_{3} & =\frac{A-j Z_{0} \sin (\beta l) \cos (\beta l) Y_{2}-\cos ^{2}(\beta l)+\sin ^{2}(\beta l)}{2 j Z_{0} \sin (\beta l) \cos (\beta l)-Z_{0}^{2} \sin ^{2}(\beta l) Y_{2}}
\end{aligned}
$$

where $Z_{0}, \beta$, and $l$ are the characteristic impedance, propagation constant, and thickness of the substrate layers, respectively. Since $A$ and $D$ are purely real and $B$ and $C$ are purely imaginary and noting that $Z_{0}$ and $\beta$ are purely real (assuming a lossless substrate), we will have purely imaginary $Y_{1}, Y_{2}$ and $Y_{3}$. Therefore, from a circuit point of view, $Y_{1}, Y_{2}$ and $Y_{3}$ represent either capacitive or inductive elements. In the next section, we calculate $Y_{1}, Y_{2}$ and $Y_{3}$ for each unit cell on each metasurface, and subsequently simulate our cascaded metasurface system in ANSYS HFSS.

\section{Full-Wave Simulated Examples}

The examples presented here use ANSYS HFSS to simulate the cascaded metasurfaces designed using the proposed procedure along with the three-layer admittance sheet topology for each unit cell.

\section{A. Phaseless Far-Field Power Pattern}

In the first example we design a pair of metasurfaces to produce a desired FF power pattern from a normally incident $\mathrm{TE}_{z}$ plane wave (i.e., only $E_{x}, E_{y}$, and $H_{z}$ field components)

\footnotetext{
${ }^{10}$ Similar relations can also be found in the Supplemental Material of [23].
} 


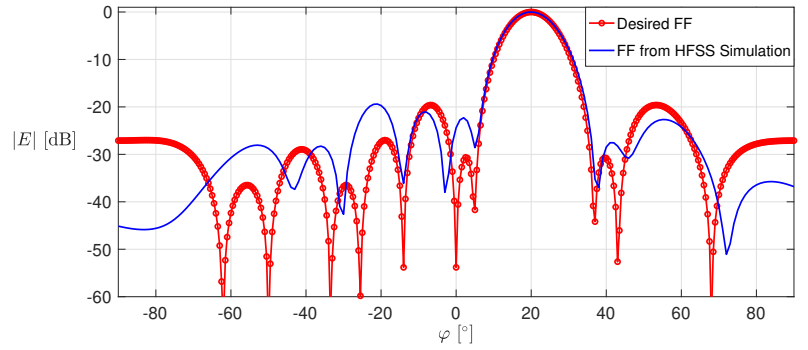

Fig. 5. Comparison of the specified desired far-field power pattern (solid red curve with circular markers) and the normalized far-field power pattern produced by the ANSYS HFSS simulation of the designed cascaded metasurfaces (solid blue curve).

at $10.5 \mathrm{GHz}$. We assume 2D field propagation in the $x y$ plane with 1D metasurfaces placed along $x=0$ and $x=-1.5 \lambda$, where $\lambda$ denotes the free space wavelength. The metasurfaces are both $5 \lambda$ in length and each metasurface is discretized into 30 unit cells of width $\lambda / 6$. While the normally incident plane wave requires periodic boundaries (implemented through the use of master/slave boundaries in HFSS), the designed metasurface will actually be aperiodic. To accommodate the simulation of an aperiodic structure within periodic boundary conditions, we add absorbing elements in-line with both metasurfaces that extend out a distance of two wavelengths from either end. These absorbers ensure that the fields close to the metasurfaces are not affected by the adjacent "images" of the structure due to the periodic boundaries.

The field specifications consist of the phaseless FF power pattern shown in Figure 5, specified for the angular range $-90^{\circ} \leq \varphi \leq 90^{\circ}$. In the first optimization step, we use $w_{\mathrm{TV}}=10^{-12}$ and a step length of 100 and minimize (12) to determine the equivalent currents $\mathbf{x}_{2}$ on the output metasurface located at $x=0$. The tangential output fields are then computed and scaled to match the total incident field power using (1) and (22). The second optimization step is then performed to minimize (23). The weighting parameters used in this step are $w_{1}=0.5, w_{2}=0.7$, and $w_{\mathrm{TV}}=8 \times 10^{-10}$, and a step length of 1 is used. Once $\mathbf{x}_{1}$ is determined, the remaining tangential fields are found using (2) and (3) and the susceptibility profiles can be computed using (33).

In this design, we use the Rogers RO3010 substrate $\left(\epsilon_{r}=\right.$ $10.2, \tan \delta=0.0022)^{11}$ for each of the two layers, each with a thickness of 50 mil. We now calculate the admittance sheet values $Y_{1}, Y_{2}$, and $Y_{3}$ for each unit cell using (35) through (37). The admittance sheets are then implemented in the HFSS simulation using impedance boundary conditions, with metallic baffles placed between each the unit cells as in [14]. The real part (absolute value) of the total electric field in the simulation domain is shown in Figure 6. The FF pattern generated from this simulation (using a NF to FF transformation) is shown in Figure 5, in which the simulated FF pattern exhibits excellent agreement with the desired FF

\footnotetext{
${ }^{11}$ Since this loss tangent is small, it has not been taken into account in the calculation of the required admittances for each unit cell of each metasurface (Section IV-I). However, the presence of this loss tangent has been taken into account in ANSYS HFSS simulations.
}

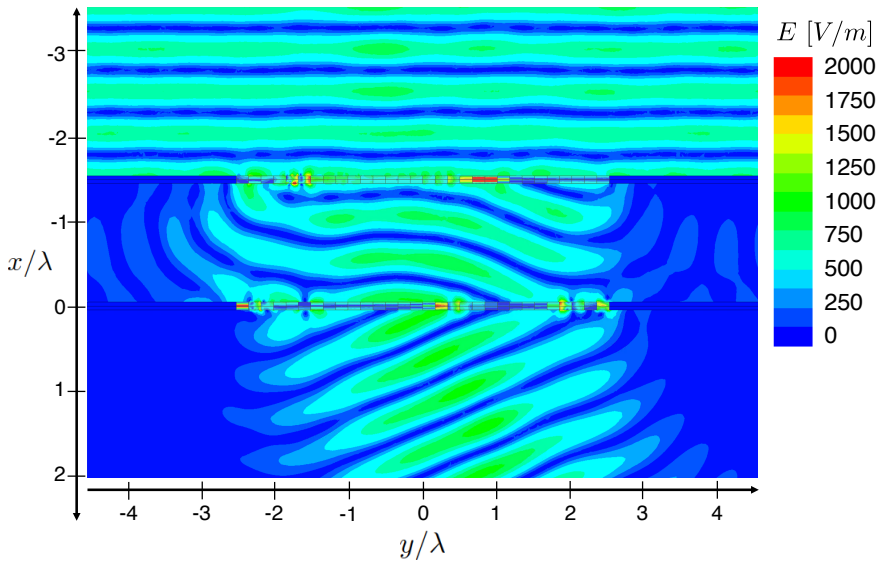

Fig. 6. Absolute value of the real part of the total electric field when the cascaded metasurface structure designed for the far-field power pattern in Figure 5 is illuminated by a normally incident plane wave.

TABLE I

Desired FAR-FIELd (FF) PERformance CRITERIA

\begin{tabular}{l|l|l} 
Specifications & Main Beam 1 & Main Beam 2 \\
\hline Direction & $\varphi=-34^{\circ}$ & $\varphi=34^{\circ}$ \\
HPBW & $16^{\circ}$ & $16^{\circ}$ \\
Nulls (relative to main beam) & $22^{\circ}$ and $38^{\circ}$ & $22^{\circ}$ and $38^{\circ}$
\end{tabular}

pattern within the main beam. Although the generated sidelobes are not exactly matched with the desired sidelobes, the generated sidelobe level (SLL) remains almost below $-20 \mathrm{~dB}$. The overall transmission efficiency, which we define as the ratio between the total real power of the output field to the total real power of the incident field, is $77.76 \%$ in this example.

\section{B. Far-Field Performance Criteria}

In the second example, the goal is to design a cascaded metasurface structure that produces a FF pattern that satisfies a collection of performance criteria. The only change required to support this type of field specifications is to replace the functional in (9) with

$$
\mathcal{C}_{\mathrm{FP}}\left(\mathbf{x}_{2}\right)=\frac{\left\|\mathbf{W}\left|\mathbf{A}_{\mathrm{F}} \mathbf{x}_{2}\right|^{2}-\mathbf{W}|\mathbf{f}|^{2}\right\|_{S}^{2}}{\left\|\mathbf{W}|\mathbf{f}|^{2}\right\|_{S}^{2}}
$$

where $\mathbf{W}$ is a diagonal prescaling matrix used to balance the relative contributions of the various performance criteria, calculated as in [17]. The details on converting the FF performance criteria into $|\mathbf{f}|^{2}$ can also be found in [17]. For this example the target FF performance criteria are shown in Table I, which consist of two identical main beams (in different directions), each with the same HPBW and associated nulls. A visual representation of these performance criteria can be seen in Figure 7, in which the elements of $\mathbf{f}$ are plotted using red circular markers. In particular, note the eight red circular markers on the $-60 \mathrm{~dB}$ level that indicates the desired eight null locations.

We adopt the same geometry and incident field as the previous example in Section V-A. In the first optimization 


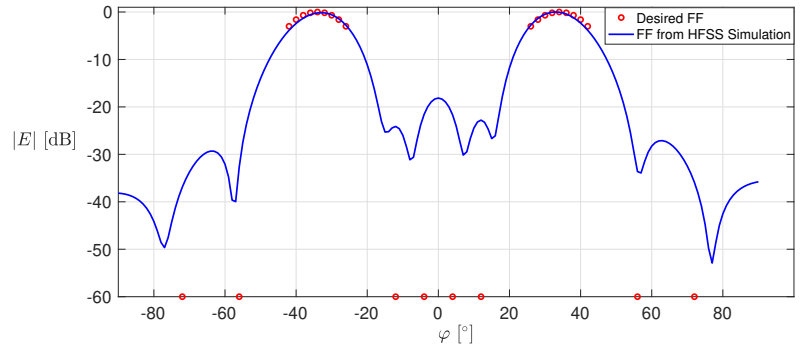

Fig. 7. Comparison of the specified far-field performance criteria (red circular markers) and the normalized far-field power pattern produced by the ANSYS HFSS simulation of the designed cascaded metasurfaces (solid blue curve). The red circular markers on the $-60 \mathrm{~dB}$ line specify the desired null locations.

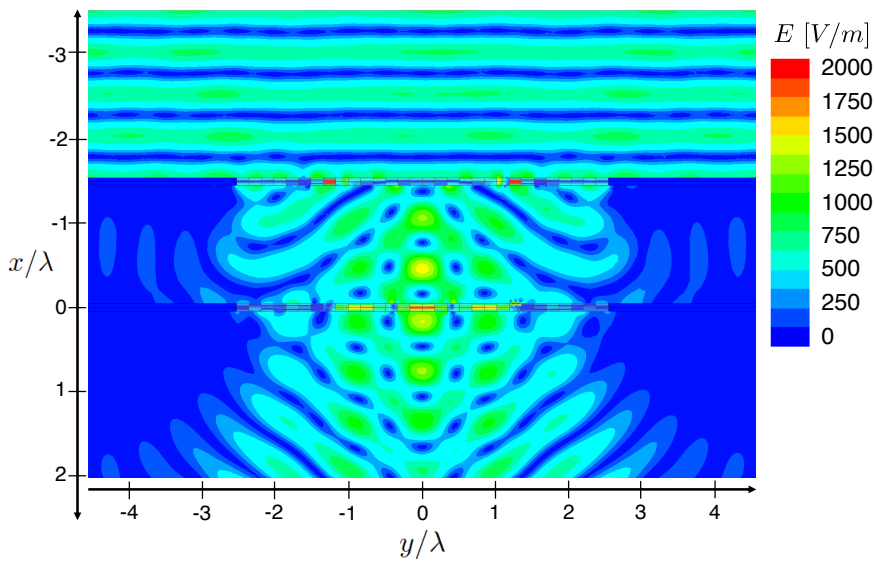

Fig. 8. Absolute value of the real part of the total electric field when the cascaded metasurface structure designed for the far-field performance criteria of Table I is illuminated by a normally incident plane wave.

step, we use parameters of $w_{\mathrm{TV}}=10^{-9}$ and a step length of 10 , and in the second optimization step we use weighting parameters $w_{1}=0.5, w_{2}=0.7, w_{\mathrm{TV}}=8 \times 10^{-10}$, and a step length of 0.1 . The real part (absolute value) of the total electric field resulting from simulating the designed metasurfaces in HFSS is shown in Figure 8, while the resulting FF is shown in Figure 7. The produced FF pattern has two main beams at $\varphi= \pm 33^{\circ}$ with HPBWs of $15.5^{\circ}$ and $15.2^{\circ}$, exhibiting excellent agreement with the field specifications. The eight desired pattern nulls are also visible, although they deviate from the intended directions by up to $5^{\circ}$. In addition, although we did not achieve very deep nulls (set to $-60 \mathrm{~dB}$ level in the specifications), all the generated nulls are still below $-20 \mathrm{~dB}$ with the best null being below $-50 \mathrm{~dB}$. Finally, the transmission efficiency observed in this case is $83.31 \%$.

\section{Comparison to a Single Metasurface with $\mathrm{TM}_{z}$ Fields}

In this final example, we change the incident field to that of an electric current line source (infinite in the $z$ direction), placed $\lambda / 3$ away from the input metasurface. The fields produced by this source are $\mathrm{TM}_{z}$ (i.e., $E_{z}, H_{x}$, and $H_{y}$ only). The frequency remains at $10.5 \mathrm{GHz}$, but each metasurface now consists of 50 unit cells of $\lambda / 10$ width and the thickness of the Rogers RO3010 substrate has been decreased to 10 mil. Absorbing elements and periodic boundary conditions are not

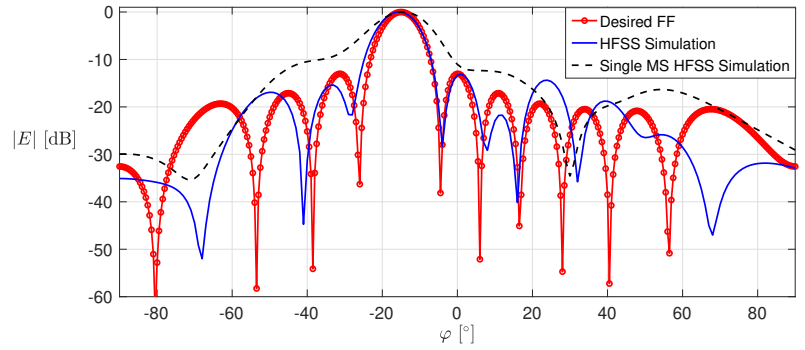

Fig. 9. Comparison of the specified far-field power pattern (solid red curve with circular markers) and the normalized far-field power patterns produced by the ANSYS HFSS simulations of the designed cascaded metasurface structure (solid blue curve) and the single metasurface (dashed black curve).

needed with this incident field, and so the simulation domain is bounded by an appropriate perfectly-matched layer (PML). ${ }^{12}$

The field specifications consist of a FF power pattern with a main beam at $\varphi=-15^{\circ}$ as shown in Figure 9. We use the proposed procedure to design a cascaded metasurface structure, with $w_{\mathrm{TV}}=10^{-5}$ and a step length of 0.005 in the first optimization step. In the second optimization step we use $w_{1}=25, w_{2}=25$, and $w_{\mathrm{TV}}=5 \times 10^{-11}$, and a step length of 1 . Additionally, we attempt to design a single metasurface using the procedure in [18] to produce a satisfactory FF pattern from the same incident field. The real part (absolute value) of the total magnetic field in the simulation domains for the single metasurface and the cascaded metasurface system are shown in Figures 10(a) and (b), respectively. In addition, the corresponding FF patterns for the single metasurface (dashed black curve) and cascaded metasurface system (solid blue curve) are shown in Figure 9. While the single metasurface has a higher transmission efficiency ( $88.00 \%$ compared to $72.36 \%$ ), the FF pattern produced by the cascaded metasurfaces is significantly closer to the target pattern. The narrow beamwidth requires a rather uniform field amplitude distributed over a large aperture, which a single metasurface is unable to produce because it is constrained by the focused input power distribution generated by the line source. In the cascaded scenario, the first metasurface is able to 'redirect' the power such that the second metasurface can more easily create the intended output field.

\section{LIMITATIONS}

The main limitation of the proposed design method is that we have not yet taken reflections between the two metasurfaces into account, and currently require that the separation be large enough such that reflections can be neglected. However, incorporating reflections into the model can allow for smaller separation between the metasurfaces and improve the transmission efficiency, as shown in [21] and [22]. A secondary limitation is that the CG step length and functional weighting parameters are determined in an ad-hoc fashion, and require some tuning to ensure convergence.

\footnotetext{
${ }^{12}$ Periodic master/slave boundaries are still present on the faces perpendicular to the $z$ axis due to the 2D nature of the simulation.
} 


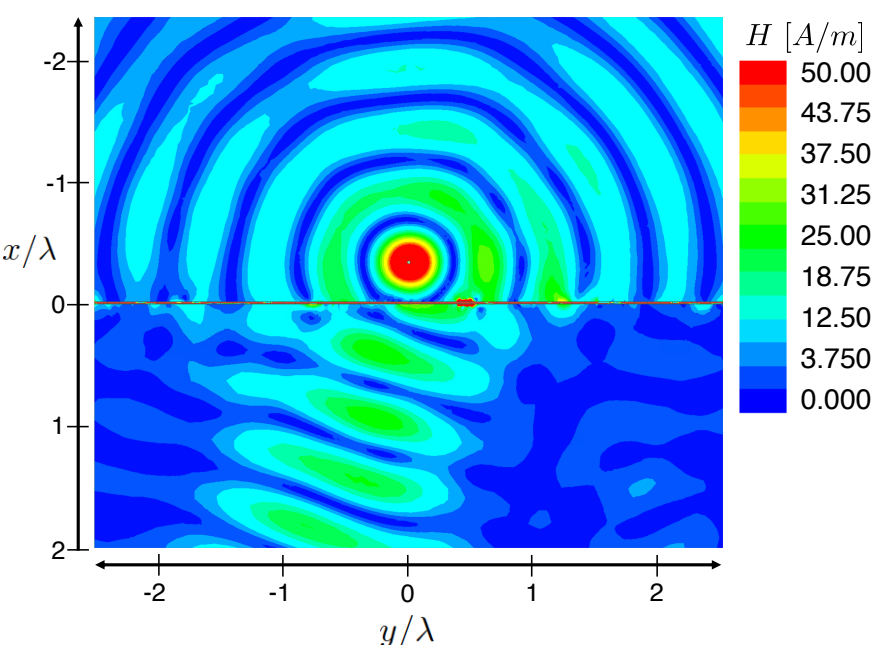

(a)

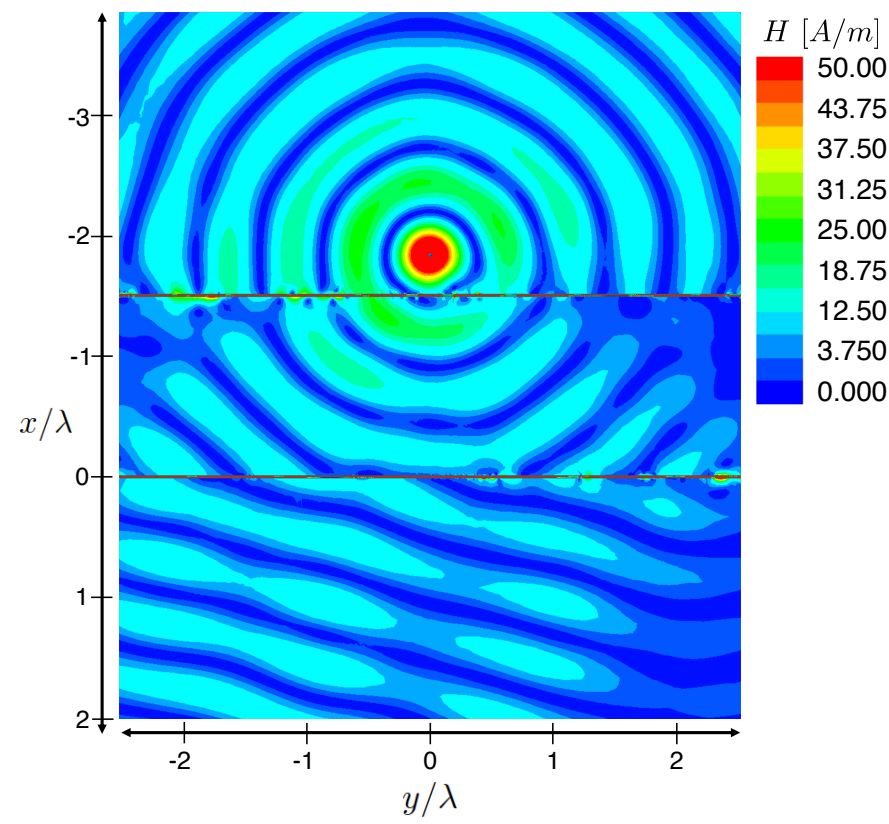

(b)

Fig. 10. Absolute value of the real part of the total magnetic field of the HFSS simulations using (a) a single metasurface and (b) two metasurfaces to produce the desired far-field power pattern shown in Figure 9 with an incident field produced by an electric current line source.

\section{CONCLUSION}

A framework for the design of passive, lossless, and reciprocal cascaded metasurfaces structures was presented. The framework utilizes electromagnetic inversion to allow for the flexibility to specify the desired output field in a variety of ways, including complex fields (amplitude and phase), phaseless fields (amplitude-only), or even in terms of far-field performance criteria such as main beam directions, half-power beamwidths, and null locations. This framework allows for full generality with respect to metasurface geometry and the locations at which the field specifications are defined. Local power conservation is enforced at each metasurface during the two-step optimization procedure, and we have introduced a total variation regularizer to improve the continuity of the resulting fields from unit cell to unit cell. Several designed metasurfaces were simulated in ANSYS HFSS using a threelayer admittance sheet topology to demonstrate the validity and flexibility of the method.

The next logical step is to physically implement the designed metasurfaces using metallic 'dogbone' structures [14], [15] or some other unit cell design. The main challenge concerning the physical implementation is compensating for the mutual coupling between the layers of each unit cell. To this end, an iterative method for tuning unit cells to account for mutual coupling was recently presented in [39]. However, a method of this nature may not be practical for the large number of unique unit cells required for the aperiodic designs presented here.

\section{APPENDIX A \\ DERIVATION OF THE REQUIRED GRADIENTS WITH RESPECT TO $\mathbf{x}_{2}$}

The CG minimization to update $\mathbf{x}_{2}$ requires finding the gradient of two cost functionals, namely, $\mathcal{C}_{\mathrm{F}}\left(\mathbf{x}_{2}\right)$ and $\mathcal{C}_{\mathrm{TV}}\left(\mathrm{x}_{2}\right)$, with respect to $\mathbf{x}_{2}$. Keep in mind that although $\mathbf{x}_{2}$ vector represents the complex-valued equivalent electric and magnetic currents on $\Sigma_{2}^{+}$, it has been constructed such that it is a purely-real vector; see Section IV-A. Therefore, all the gradient vectors will also be purely real vectors.

\section{A. Gradient of $\mathcal{C}_{F}\left(\mathbf{x}_{2}\right)$}

We begin deriving the gradient of $\mathcal{C}_{\mathrm{F}}\left(\mathrm{x}_{2}\right)$ using standard vector differentiation rules as

$$
\begin{aligned}
\mathbf{g}_{\mathrm{F}}\left(\mathbf{x}_{2}\right) & =\frac{\partial}{\partial \mathbf{x}_{2}} \frac{\left\|\left|\mathbf{A}_{\mathrm{F}} \mathbf{x}_{2}\right|^{2}-|\mathbf{f}|^{2}\right\|_{S}^{2}}{\left\||\mathbf{f}|^{2}\right\|_{S}^{2}} \\
& =\frac{2}{\left\||\mathbf{f}|^{2}\right\|_{S}^{2}} \frac{\partial}{\partial \mathbf{x}_{2}}\left[\left(\mathbf{A}_{\mathrm{F}} \mathbf{x}_{2}\right) \odot\left(\mathbf{A}_{\mathrm{F}} \mathbf{x}_{2}\right)^{*}-|\mathbf{f}|^{2}\right]^{\mathrm{T}} \mathbf{r}_{\mathrm{F}}
\end{aligned}
$$

where $\mathbf{r}_{\mathrm{F}}$ is given in (16) and $\odot$ represents the Hadamard (elementwise) product. To evaluate the derivative in (39), we use the identity

$$
\frac{\partial}{\partial \mathbf{x}}(\mathbf{A x} \odot \mathbf{B} \mathbf{x})=\operatorname{diag}(\mathbf{A x}) \mathbf{B}+\operatorname{diag}(\mathbf{B} \mathbf{x}) \mathbf{A} .
$$

Noting that $\mathbf{x}_{2}$ is a purely real vector $\left(\mathbf{x}_{2} \in \mathbb{R}^{4 N}\right)$, the use of (40) in conjunction with (39) results in

$$
\begin{aligned}
\mathbf{g}_{\mathrm{F}}\left(\mathbf{x}_{2}\right) & =\frac{2}{\left\||\mathbf{f}|^{2}\right\|_{S}^{2}}\left[\operatorname{diag}\left(\mathbf{A}_{\mathrm{F}} \mathbf{x}_{2}\right) \mathbf{A}_{\mathrm{F}}^{*}+\operatorname{diag}\left(\mathbf{A}_{\mathrm{F}}^{*} \mathbf{x}_{2}\right) \mathbf{A}_{\mathrm{F}}\right]^{\mathrm{T}} \mathbf{r}_{\mathrm{F}} \\
& =\frac{2}{\left\||\mathbf{f}|^{2}\right\|_{S}^{2}}\left[2 \operatorname{Re}\left\{\operatorname{diag}\left(\mathbf{A}_{\mathrm{F}} \mathbf{x}_{2}\right) \mathbf{A}_{\mathrm{F}}^{*}\right\}\right]^{\mathrm{T}} \mathbf{r}_{\mathrm{F}}
\end{aligned}
$$


Noting that the residual $\mathbf{r}_{\mathrm{F}}$ is purely real and applying the transpose operation we can simplify this expression to

$$
\begin{aligned}
\mathbf{g}_{\mathrm{F}}\left(\mathbf{x}_{2}\right) & =\frac{4 \operatorname{Re}\left\{\mathbf{A}_{\mathrm{F}}^{\mathrm{H}} \operatorname{diag}\left(\mathbf{A}_{\mathrm{F}} \mathbf{x}_{2}\right) \mathbf{r}_{\mathrm{F}}\right\}}{\left\||\mathbf{f}|^{2}\right\|_{S}^{2}} \\
& =\frac{4 \operatorname{Re}\left\{\mathbf{A}_{\mathrm{F}}^{\mathrm{H}}\left(\mathbf{r}_{\mathrm{F}} \odot \mathbf{A}_{\mathrm{F}} \mathbf{x}_{2}\right)\right\}}{\left\||\mathbf{f}|^{2}\right\|_{S}^{2}}
\end{aligned}
$$

\section{B. Gradient of $\mathcal{C}_{T V}\left(\mathrm{x}_{2}\right)$}

Next, consider the gradient of the total variation regularizer given in (10). As an analogous derivation, we instead consider the functional

$$
\tilde{\mathcal{C}}_{\mathrm{TV}}(u(y))=\left\|\nabla_{y} u(y)\right\|_{\Sigma_{2}}^{2}
$$

in which $u(y)$ is a continuously defined real-valued function of $y$ on $\Sigma_{2}$ and the norm is defined as

$$
\|\vec{f}(y)\|_{\Sigma_{2}}^{2}=\langle\vec{f}(y), \vec{f}(y)\rangle_{\Sigma_{2}}=\int_{\Sigma_{2}} \vec{f}(y) \cdot \vec{f}(y) d y .
$$

Note that since we are dealing with real-valued functions (i.e., the continuous form of the real-valued $\mathrm{x}_{2}$ vector), the presence of the complex conjugate operator has been dropped in the above norm definition. ${ }^{13}$ For the sake of notational simplicity, the $y$ dependency of $u$ will be implied from now on. We start by finding the first variation of $\tilde{\mathcal{C}}_{\mathrm{TV}}(u)$, i.e., the derivative of $\tilde{\mathcal{C}}_{\mathrm{TV}}(u)$ with respect to $u$ when $u$ is slightly varied by some function $\psi$ (which is defined on the same domain as $u$ )

$$
\begin{aligned}
\partial \tilde{\mathcal{C}}_{\mathrm{TV}} & =\lim _{\varepsilon \rightarrow 0} \frac{\tilde{\mathcal{C}}_{\mathrm{TV}}(u+\varepsilon \psi)-\tilde{\mathcal{C}}_{\mathrm{TV}}(u)}{\varepsilon} \\
& =\lim _{\varepsilon \rightarrow 0} \frac{\left\|\nabla_{y}(u+\varepsilon \psi)\right\|_{\Sigma_{2}}^{2}-\left\|\nabla_{y} u\right\|_{\Sigma_{2}}^{2}}{\varepsilon} .
\end{aligned}
$$

Using the definition of the norm in (44), we can expand (45) as

$$
\begin{aligned}
\partial \tilde{\mathcal{C}}_{\mathrm{TV}} & =\lim _{\varepsilon \rightarrow 0} \frac{\left\langle\nabla_{y} u+\varepsilon \nabla_{y} \psi, \nabla_{y} u+\varepsilon \nabla_{y} \psi\right\rangle-\left\|\nabla_{y} u\right\|_{\Sigma_{2}}^{2}}{\varepsilon} \\
& =\lim _{\varepsilon \rightarrow 0} \frac{\varepsilon^{2}\left\|\nabla_{y} \psi\right\|_{\Sigma_{2}}^{2}+2\left\langle\nabla_{y} u, \varepsilon \nabla_{y} \psi\right\rangle}{\varepsilon}
\end{aligned}
$$

and evaluating the limit results in

$$
\begin{aligned}
\partial \tilde{\mathcal{C}}_{\mathrm{TV}} & =2\left\langle\nabla_{y} u, \nabla_{y} \psi\right\rangle \\
& =2 \int_{\Sigma_{2}}\left(\nabla_{y} u\right) \cdot\left(\nabla_{y} \psi\right) d y .
\end{aligned}
$$

If we note that

$$
\nabla_{y} \cdot\left(\left(\nabla_{y} u\right) \psi\right)=\psi \nabla_{y} \cdot \nabla_{y} u+\nabla_{y} u \cdot \nabla_{y} \psi
$$

then (47) becomes

$$
\partial \tilde{\mathcal{C}}_{\mathrm{TV}}=2 \int_{\Sigma_{2}}\left[\nabla_{y} \cdot\left(\left(\nabla_{y} u\right) \psi\right)-\psi \nabla_{y} \cdot \nabla_{y} u\right] d y .
$$

\footnotetext{
${ }^{13}$ For a similar derivation, but in the complex domain, see [40, Appendix D.3].
}

Applying the divergence theorem [41] results in

$$
\partial \tilde{\mathcal{C}}_{\mathrm{TV}}=2 \int_{\partial \Sigma_{2}}\left(\nabla_{y} u\right) \psi \cdot \hat{x} d y-2 \int_{\Sigma_{2}} \psi \nabla_{y} \cdot\left(\nabla_{y} u\right) d y
$$

where $\partial \Sigma_{2}$ refers to the edges of the metasurface. We assume that the function $u$ vanishes on the boundary, i.e., $u\left(y \in \partial \Sigma_{2}\right)=0$, which is equivalent to assuming that the equivalent currents are assumed to be zero on the edges of the metasurface in our case. Since $\psi$ exists in the same function space as $u$, then $\psi\left(y \in \partial \Sigma_{2}\right)=0$. Subsequently, (50) simplifies to

$$
\begin{aligned}
\partial \tilde{\mathcal{C}}_{\mathrm{TV}} & =-2 \int_{\Sigma_{2}} \psi \nabla_{y} \cdot\left(\nabla_{y} u\right) d y \\
& =\left\langle-2 \nabla_{y} \cdot\left(\nabla_{y} u\right), \psi\right\rangle_{\Sigma_{2}} \\
& =\left\langle-2 \nabla_{y}^{2} u, \psi\right\rangle_{\Sigma_{2}} .
\end{aligned}
$$

If we were to discretize (43) such that the functional operates on the equivalent currents in $\mathbf{x}_{2}$ as in (10), and considering the regularization weight $w_{\mathrm{TV}}$, the corresponding discrete gradient operator [42], [43] can then be written as

$$
\mathbf{g}_{\mathrm{TV}}\left(\mathbf{x}_{2}\right)=-2 w_{\mathrm{TV}}\left[\begin{array}{c}
\frac{\partial^{2}}{\partial y^{2}} \mathbf{J}_{2, \mathrm{R}} \\
\frac{\partial^{2}}{\partial y^{2}} \mathbf{J}_{2, \mathrm{I}} \\
\frac{\partial^{2}}{\partial y^{2}} \mathbf{M}_{2, \mathrm{R}} \\
\frac{\partial^{2}}{\partial y^{2}} \mathbf{M}_{2, \mathrm{I}}
\end{array}\right] .
$$

\section{APPENDIX B \\ DERIVATION OF THE REQUIRED GRADIENTS WITH RESPECT TO $\mathbf{x}_{1}$}

This requires calculation of the gradient of the four cost functionals, namely $\mathcal{C}_{\mathrm{L}}, \mathcal{C}_{\mathrm{P}, 1}, \mathcal{C}_{\mathrm{P}, 2}$, and $\mathcal{C}_{\mathrm{TV}}$, with respect to $\mathbf{x}_{1}$. Keep in mind that although $\mathbf{x}_{1}$ represents the complex-valued equivalent electric and magnetic currents on $\Sigma_{1}^{+}$, it has been constructed to be a purely real vector; see Section IV-A.

\section{A. Gradient of $\mathcal{C}_{L}\left(\mathbf{x}_{1}\right)$}

The gradient of $\mathcal{C}_{\mathrm{L}}\left(\mathbf{x}_{1}\right)$ can be derived using vector differentiation rules as

$$
\begin{aligned}
\mathbf{g}_{\mathrm{L}} & =\frac{\partial}{\partial \mathbf{x}_{1}}\left\|\mathbf{L} \mathbf{x}_{1}\right\|_{\Sigma_{1}}^{2} \\
& =\frac{\partial}{\partial \mathbf{x}_{1}}\left[\left(\mathbf{L} \mathbf{x}_{1}\right)^{\mathrm{H}}\left(\mathbf{L} \mathbf{x}_{1}\right)\right] \\
& =\frac{\partial}{\partial \mathbf{x}_{1}}\left[\mathbf{x}_{1}^{\mathrm{T}} \mathbf{L}^{\mathrm{H}} \mathbf{L} \mathbf{x}_{1}\right] \\
& =\left[\mathbf{L}^{\mathrm{H}} \mathbf{L}+\left(\mathbf{L}^{\mathrm{H}} \mathbf{L}\right)^{\mathrm{T}}\right] \mathbf{x}_{1} \\
& =\left(\mathbf{L}^{\mathrm{H}} \mathbf{L}+\mathbf{L}^{\mathrm{T}} \mathbf{L}^{*}\right) \mathbf{x}_{1} \\
& =2 \operatorname{Re}\left(\mathbf{L}^{\mathrm{H}} \mathbf{L} \mathbf{x}_{1}\right)
\end{aligned}
$$




\section{B. Gradient of $\mathcal{C}_{P, I}\left(\mathbf{x}_{1}\right)$}

To derive the required gradient of $\mathcal{C}_{\mathrm{P}, 1}\left(\mathrm{x}_{1}\right)$, we first find the gradient of $\mathcal{C}_{\mathrm{P}, 1}\left(\mathbf{x}_{1}\right)$ with respect to $\mathbf{J}_{1, \mathrm{R}}$ only. Using vector differentiation rules this can be computed as

$$
\begin{aligned}
\frac{\partial \mathcal{C}_{\mathrm{P}, 1}}{\partial \mathbf{J}_{1, \mathrm{R}}} & =\frac{\partial}{\partial \mathbf{J}_{1, \mathrm{R}}} \frac{w_{1}\left\|\frac{1}{2}\left(\mathbf{J}_{1, \mathrm{R}} \odot \mathbf{M}_{1, \mathrm{R}}+\mathbf{J}_{1, \mathrm{I}} \odot \mathbf{M}_{1, \mathrm{I}}\right)-\mathbf{p}_{\text {in }}\right\|_{\Sigma_{1}}^{2}}{\left\|\mathbf{p}_{\text {in }}\right\|_{\Sigma_{1}}^{2}} \\
& =\frac{2 w_{1} \frac{\partial}{\partial \mathbf{J}_{1, \mathrm{R}}}\left[\frac{1}{2}\left(\mathbf{J}_{1, \mathrm{R}} \odot \mathbf{M}_{1, \mathrm{R}}+\mathbf{J}_{1, \mathrm{I}} \odot \mathbf{M}_{1, \mathrm{I}}\right)-\mathbf{p}_{\text {in }}\right]^{\mathrm{T}} \mathbf{r}_{1}}{\left\|\mathbf{p}_{\text {in }}\right\|_{\Sigma_{1}}^{2}} \\
& =\frac{w_{1}}{\left\|\mathbf{p}_{\text {in }}\right\|_{\Sigma_{1}}^{2}} \operatorname{diag}\left(\mathbf{M}_{1, \mathrm{R}}\right) \mathbf{r}_{1} \\
& =\frac{w_{1}}{\left\|\mathbf{p}_{\text {in }}\right\|_{\Sigma_{1}}^{2}} \mathbf{r}_{1} \odot \mathbf{M}_{1, \mathrm{R}}
\end{aligned}
$$

where $\mathbf{r}_{1}$ is given in (30). Performing the a similar derivation for the gradients with respect to $\mathbf{J}_{1, \mathrm{I}}, \mathbf{M}_{1, \mathrm{R}}$, and $\mathbf{M}_{1, \mathrm{I}}$ and concatenating the results according to the order of (8) produces the final gradient with respect to $\mathbf{x}_{1}$, i.e., $\mathbf{g}_{\mathrm{P}, 1}$, which is given in (29).

\section{Gradient of $\mathcal{C}_{P, 2}\left(\mathbf{x}_{1}\right)$}

We now derive the gradient of $\mathcal{C}_{\mathrm{P}, 2}\left(\mathbf{x}_{1}\right)$ as

$$
\begin{aligned}
\mathbf{g}_{\mathrm{P}, 2} & =\frac{\partial}{\partial \mathbf{x}_{1}} \frac{w_{2}}{\left\|\frac{1}{2} \operatorname{Re}\left(\mathbf{B}_{1} \mathbf{x}_{1} \odot \mathbf{B}_{2}^{*} \mathbf{x}_{1}\right)-\mathbf{p}_{\text {out }}\right\|_{\Sigma_{2}}^{2}} \\
& =\frac{2 w_{2} \frac{\partial}{\partial \mathbf{x}_{1}}\left[\frac{1}{2} \operatorname{Re}\left(\mathbf{B}_{1} \mathbf{x}_{1} \odot \mathbf{B}_{2}^{*} \|_{\Sigma_{2}}^{2} \mathbf{x}_{1}\right)-\mathbf{p}_{\text {out }}\right]^{\mathrm{T}} \mathbf{r}_{2}}{\left\|\mathbf{p}_{\text {out }}\right\|_{\Sigma_{2}}^{2}} .
\end{aligned}
$$

Using the identity in (40) this simplifies to

$$
\mathbf{g}_{\mathrm{P}, 2}=\frac{w_{2}\left[\operatorname{Re}\left(\operatorname{diag}\left(\mathbf{B}_{1} \mathbf{x}_{1}\right) \mathbf{B}_{2}^{*}+\operatorname{diag}\left(\mathbf{B}_{2}^{*} \mathbf{x}_{1}\right) \mathbf{B}_{1}\right)\right]^{\mathrm{T}} \mathbf{r}_{2}}{\left\|\mathbf{p}_{\text {out }}\right\|_{\Sigma_{2}}^{2}} .
$$

\section{Gradient of $\mathcal{C}_{T V}\left(\mathbf{x}_{1}\right)$}

This is the same as Section A-B. Thus,

$$
\mathbf{g}_{\mathrm{TV}}\left(\mathbf{x}_{1}\right)=-2 w_{\mathrm{TV}}\left[\begin{array}{c}
\frac{\partial^{2}}{\partial y^{2}} \mathbf{J}_{1, \mathrm{R}} \\
\frac{\partial^{2}}{\partial y^{2}} \mathbf{J}_{1, \mathrm{I}} \\
\frac{\partial^{2}}{\partial y^{2}} \mathbf{M}_{1, \mathrm{R}} \\
\frac{\partial^{2}}{\partial y^{2}} \mathbf{M}_{1, \mathrm{I}}
\end{array}\right] .
$$

\section{ACKNOWLEDGMENT}

The authors would like to thank the Canadian Microelectronics Corp. for the provision of ANSYS HFSS. The financial support of the Natural Sciences and Engineering Research Council (NSERC) of Canada and the Canada Research Chair (CRC) Program is also acknowledged.

\section{REFERENCES}

[1] C. L. Holloway, E. F. Kuester, J. A. Gordon, J. O'Hara, J. Booth, and D. R. Smith, "An overview of the theory and applications of metasurfaces: The two-dimensional equivalents of metamaterials," IEEE Antennas and Propagation Magazine, vol. 54, no. 2, pp. 10-35, 2012.

[2] M. Selvanayagam and G. Eleftheriades, "Discontinuous electromagnetic fields using orthogonal electric and magnetic currents for wavefront manipulation," Optics Express, pp. 14 409-14429, 2013.

[3] C. Pfeiffer and A. Grbic, "Metamaterial Huygens' surfaces: Tailoring wave fronts with reflectionless sheets," Phys. Rev. Lett., vol. 110, p. 197401, May 2013.

[4] G. Minatti, M. Faenzi, E. Martini, F. Caminita, P. De Vita, D. GonzálezOvejero, M. Sabbadini, and S. Maci, "Modulated metasurface antennas for space: Synthesis, analysis and realizations," IEEE Transactions on Antennas and Propagation, vol. 63, no. 4, pp. 1288-1300, April 2015.

[5] S. Tretyakov, "Metasurfaces for general transformations of electromagnetic fields," Philos. Trans. Royal Soc. A: Mathematical, Physical and Engineering Sciences, vol. 373, no. 2049, p. 20140362, 2015.

[6] K. Achouri and C. Caloz, "Design, concepts and applications of electromagnetic metasurfaces," Nanophotonics, vol. 7, no. 6, pp. 1095-1116, 2018.

[7] A. H. Dorrah and G. V. Eleftheriades, "Bianisotropic Huygens' metasurface pairs for nonlocal power-conserving wave transformations," IEEE Antennas and Wireless Propagation Letters, vol. 17, no. 10, pp. 17881792 , Oct 2018

[8] C. Pfeiffer and A. Grbic, "Bianisotropic metasurfaces for optimal polarization control: Analysis and synthesis," Physical Review Applied, vol. 2, no. 4, p. 044011, 2014.

[9] M. Selvanayagam and G. V. Eleftheriades, "Polarization control using tensor Huygens surfaces," IEEE Transactions on Antennas and Propagation, vol. 62, no. 12, pp. 6155-6168, 2014.

[10] A. H. Dorrah, M. Chen, and G. V. Eleftheriades, "Bianisotropic Huygens' metasurface for wideband impedance matching between two dielectric media," IEEE Transactions on Antennas and Propagation, vol. 66, no. 9, pp. 4729-4742, Sep. 2018.

[11] M. Dehmollaian, N. Chamanara, and C. Caloz, "Wave scattering by a cylindrical metasurface cavity of arbitrary cross section: Theory and applications," IEEE Transactions on Antennas and Propagation, vol. 67, no. 6, pp. 4059-4072, 2019.

[12] A. Epstein and G. V. Eleftheriades, "Huygens' metasurfaces via the equivalence principle: design and applications," Journal of the Optical Society of America B, vol. 33, no. 2, pp. A31-A50, 2016.

[13] K. Achouri, M. A. Salem, and C. Caloz, "General metasurface synthesis based on susceptibility tensors," IEEE Transactions on Antennas and Propagation, vol. 63, no. 7, pp. 2977-2991, 2015.

[14] G. Xu, S. V. Hum, and G. V. Eleftheriades, "Augmented Huygens' metasurfaces employing baffles for precise control of wave transformations," IEEE Transactions on Antennas and Propagation, vol. 67, no. 11, pp. 6935-6946, June 2019.

[15] G. Lavigne, K. Achouri, V. S. Asadchy, S. A. Tretyakov, and C. Caloz, "Susceptibility derivation and experimental demonstration of refracting metasurfaces without spurious diffraction," IEEE Transactions on Antennas and Propagation, vol. 66, no. 3, pp. 1321-1330, 2018.

[16] T. Brown, C. Narendra, and P. Mojabi, "On the use of the source reconstruction method for metasurface design," 12th European Conference on Antennas and Propagation (EuCAP), pp. 302-306, April 2018.

[17] T. Brown, C. Narendra, Y. Vahabzadeh, C. Caloz, and P. Mojabi, "On the use of electromagnetic inversion for metasurface design," IEEE Transactions on Antennas and Propagation, vol. 68, no. 3, pp. 18121824, March 2020.

[18] T. Brown, Y. Vahabzadeh, C. Caloz, and P. Mojabi, "Electromagnetic inversion with local power conservation for metasurface design," IEEE Antennas and Wireless Propagation Letters, 2020.

[19] A. Epstein and G. V. Eleftheriades, "Arbitrary power-conserving field transformations with passive lossless omega-type bianisotropic metasurfaces," IEEE Transactions on Antennas and Propagation, vol. 64, no. 9, pp. 3880-3895, 2016.

[20] — "Passive lossless Huygens metasurfaces for conversion of arbitrary source field to directive radiation," IEEE Transactions on Antennas and Propagation, vol. 62, no. 11, pp. 5680-5695, 2014.

[21] V. G. Ataloglou, A. H. Dorrah, and G. V. Eleftheriades, "Design of compact Huygens' metasurface pairs with multiple reflections for arbitrary wave transformations," IEEE Transactions on Antennas and Propagation, 2020. 
[22] _ - "Realizing antenna arrays with Huygens' metasurface pairs based on a moment-method-like design," in 2020 14th European Conference on Antennas and Propagation (EuCAP). IEEE, 2020, pp. 1-5.

[23] B. O. Raeker and A. Grbic, "Compound metaoptics for amplitude and phase control of wave fronts," Phys. Rev. Lett., vol. 122, p. 113901, Mar 2019. [Online]. Available: https://link.aps.org/doi/10.1103/PhysRevLett.122.113901

[24] B. O. Raeker, A. Grbic, Y. Zhou, and J. Valentine, "All-dielectric compound metaoptics," in 2019 IEEE International Symposium on Antennas and Propagation and USNC-URSI Radio Science Meeting, 2019, pp. 431-432.

[25] A. Abubakar, P. M. van den Berg, and J. J. Mallorqui, "Imaging of biomedical data using a multiplicative regularized contrast source inversion method," IEEE Transactions on Microwave Theory and Techniques, vol. 50, no. 7, pp. 1761-1771, 2002.

[26] P. Mojabi and J. LoVetri, "Microwave biomedical imaging using the multiplicative regularized Gauss-Newton inversion," IEEE Antennas and Wireless Propagation Letters, vol. 8, pp. 645-648, 2009.

[27] M. M. Idemen, Discontinuities in the electromagnetic field. John Wiley \& Sons (IEEE Press series on electromagnetic wave theory; 40), 2011.

[28] E. F. Kuester, M. A. Mohamed, M. Piket-May, and C. L. Holloway, "Averaged transition conditions for electromagnetic fields at a metafilm," IEEE Transactions on Antennas and Propagation, vol. 51, no. 10, pp. 2641-2651, 2003.

[29] J. L. A. Quijano and G. Vecchi, "Field and source equivalence in source reconstruction on 3D surfaces," Progress In Electromagnetics Research, vol. 103 , pp. $67-100,2010$

[30] M. Phaneuf and P. Mojabi, "Electromagnetic inversion for noninvasive specific absorption rate characterization," IEEE Journal of Electromagnetics, $R F$ and Microwaves in Medicine and Biology, 2020.

[31] T. Brown, I. Jeffrey, and P. Mojabi, "Multiplicatively regularized source reconstruction method for phaseless planar near-field antenna measurements," IEEE Transactions on Antennas and Propagation, vol. 65, no. 4, pp. 2020-2031, 2017.

[32] A. Abubakar, P. M. van den Berg, T. M. Habashy, and H. Braunisch, "A multiplicative regularization approach for deblurring problems," Image Processing, IEEE Transactions on, vol. 13, no. 11, pp. 1524-1532, 2004.

[33] E. Polak and G. Ribiere, "Note sur la convergence de méthodes de directions conjuguées," Revue française d'informatique et de recherche opérationnelle, série rouge, vol. 3, no. 1, pp. 35-43, 1969.

[34] K. Achouri, "Synthesis and applications of electromagnetic metasurfaces," Ph.D. dissertation, École Polytechnique de Montr'/eal, Montreal, Canada, 2017

[35] J. P. Wong, A. Epstein, and G. V. Eleftheriades, "Reflectionless wideangle refracting metasurfaces," IEEE Antennas and Wireless Propagation Letters, vol. 15, pp. 1293-1296, 2015.

[36] M. Chen, E. Abdo-Sánchez, A. Epstein, and G. V. Eleftheriades, "Theory, design, and experimental verification of a reflectionless bianisotropic Huygens' metasurface for wide-angle refraction," Physical Review B, vol. 97 , no. 12, p. 125433, 2018.

[37] F. Monticone, N. M. Estakhri, and A. Alù, "Full control of nanoscale optical transmission with a composite metascreen," Phys. Rev. Lett., vol. 110, p. 203903, May 2013. [Online]. Available: https://link.aps.org/doi/10.1103/PhysRevLett.110.203903

[38] T. Brown, Z. Liu, and P. Mojabi, "Full-wave verification of an electromagnetic inversion metasurface design method," TechRxiv preprint, Jan 2020.

[39] A. E. Olk and D. A. Powell, "Accurate metasurface synthesis incorporating near-field coupling effects," Physical Review Applied, vol. 11, no. 6, p. 064007, 2019.

[40] P. Mojabi, "Investigation and development of algorithms and techniques for microwave tomography," Ph.D. dissertation, University of Manitoba, Winnipeg, Manitoba, Canada (Available Online), 2010.

[41] J. D. Jackson, Classical Electrodynamics, 3rd ed. John Wiley \& Sons, 1999.

[42] D. Brandwood, "A complex gradient operator and its application in adaptive array theory," in IEE Proceedings F: Communications Radar and Signal Processing, vol. 130, 1983, pp. 11-16.

[43] A. Van Den Bos, "Complex gradient and Hessian," IEE ProceedingsVision, Image and Signal Processing, vol. 141, no. 6, pp. 380-383, 1994. 